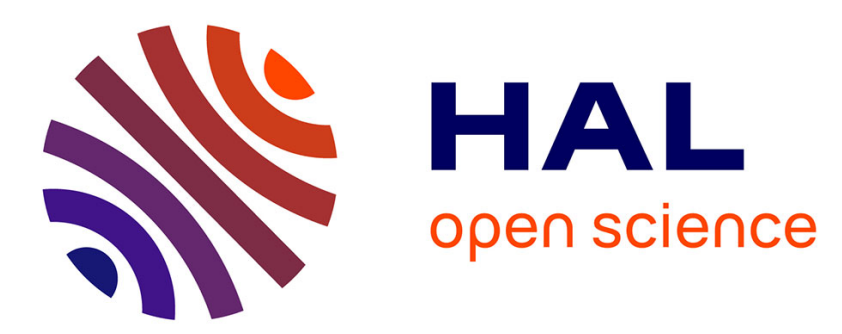

\title{
Experimental and theoretical study of iron and mild steel combustion in oxygen flows
}

\author{
Hazem El-Rabii, Kirill A. Kazakov, Maryse Muller
}

\section{To cite this version:}

Hazem El-Rabii, Kirill A. Kazakov, Maryse Muller. Experimental and theoretical study of iron and mild steel combustion in oxygen flows. Physics of Fluids, 2017, 29 (3), pp.037104. 10.1063/1.4977746 . hal-01500767

\section{HAL Id: hal-01500767 \\ https://hal.science/hal-01500767}

Submitted on 3 Apr 2017

HAL is a multi-disciplinary open access archive for the deposit and dissemination of scientific research documents, whether they are published or not. The documents may come from teaching and research institutions in France or abroad, or from public or private research centers.
L'archive ouverte pluridisciplinaire HAL, est destinée au dépôt et à la diffusion de documents scientifiques de niveau recherche, publiés ou non, émanant des établissements d'enseignement et de recherche français ou étrangers, des laboratoires publics ou privés. 


\title{
Experimental and theoretical study of iron and mild steel combustion in oxygen flows
}

\author{
Hazem El-Rabii, ${ }^{1}$ Kirill A. Kazakov, ${ }^{2}$ and Maryse Muller ${ }^{3}$ \\ ${ }^{1}$ Institut Pprime, CNRS (UPR 3346), 1 Avenue Clément Ader, 86961 Futuroscope Chasseneuil, France \\ ${ }^{2}$ Physics Faculty, Department of Theoretical Physics, Moscow State University, \\ 119991 Moscow, Russian Federation \\ ${ }^{3}$ Laboratoire PIMM, CNRS/Arts et Métiers ParisTech, 151 Boulevard de l'Hôpital, 75013 Paris, France
}

\begin{abstract}
The effects of oxygen flow speed and pressure on the iron and mild steel combustion are investigated experimentally and theoretically. The studied specimens are vertical cylindrical rods subjected to an axial oxygen flow and ignited at the upper end by laser irradiation. Three main stages of the combustion process have been identified experimentally: (1) induction period, during which the rod is heated until an intensive metal oxidation begins at its upper end; (2) static combustion, during which a laminar liquid "cap" slowly grows on the upper rod end, and, after the liquid cap detachment from the sample; (3) dynamic combustion, which is characterized by a rapid metal consumption and turbulent liquid motions. An analytical description of these stages is given. In particular, a model of the dynamic combustion is constructed based on the turbulent oxygen transport through the liquid metal-oxide flow. This model yields a simple expression for the fraction of metal burned in the process and allows one to calculate the normal propagation speed of the solid metal-liquid interface as a function of the oxygen flow speed and pressure. A comparison of the theory with the experimental results is made, and its potential application is mentioned.
\end{abstract}

\section{INTRODUCTION}

Since the 1950s, combustion of metals has attracted much attention on the account of its numerous practical applications. This interest is motivated by two important features of this process, namely, the large heat of combustion and the refractory nature of metal oxides. The research has been focused primarily on the propulsion technologies which use metallic particles as high-energy additives in propellants to increase the specific impulses and combustion stability of solid rocket motors. ${ }^{1,2}$ The refractory nature of combustion products, on the other hand, has been exploited to synthesize a wide range of materials, including (nano) powders, intermetallics, composites, and functionally graded materials. ${ }^{3-7}$ Many important results have been obtained in these directions which are still subjects of ongoing intensive experimental and theoretical studies. ${ }^{8-15}$

Yet, the large amount of energy released by a burning metal has undesirable consequences and represents a source of significant fire hazards, especially when metals are used in high-temperature and/or high-pressure oxidizing environments such as those prevailing in nuclear plants and oxygen supply systems. ${ }^{16,17}$ The research conducted in metal-fire prevention has mostly consisted of carrying out standard tests that quantify the relative flammability of different metals, that is, their relative propensity to sustain combustion of metallic materials of standardized dimensions in oxygen atmospheres. ${ }^{18}$ Surprisingly enough, no single test has been developed to date that could provide either absolute ignition limits or consistent relative ratings for all materials. The reason is probably that comparatively few efforts have been devoted to this topic of basic research which is barely covered in the literature. Specifically, theoretical attempts to understand and describe the nature of combustion of bulk metallic materials have been hardly undertaken, ${ }^{19,20}$ and to the best of our knowledge, the effect of the melt flow hydrodynamics on the burning process has never been addressed, although its importance has been highlighted. ${ }^{21,22}$

The present contribution is an effort to fill this gap. We report an experimental and theoretical investigation of initiation and progression of the metal combustion in an oxygen flow under normal and elevated pressures. The studied specimens are vertical cylindrical rods ignited by heating up their top surfaces with a laser beam. Vertical cylindrical rods ignited at the lower end are normally used by the American Society for Testing and Materials in the standard procedure of testing metal inflammability, ${ }^{18}$ whereas sample shapes other than cylindrical might be of greater importance for applications. The reason to choose our configuration is that it is most suitable for a detailed study of the metal combustion wave, in particular, for determination of its normal propagation speed with respect to the solid metal. The vertical homogeneity provided by cylindrical geometry allows us to achieve global steadiness of the wave during most part of the process, while its downward propagation leads to the formation of a well-defined interface between the solid and liquid phases, which becomes a nearly planar slope under the action of the combustion products driven along it by the imposed oxygen flow, thus greatly facilitating both the normal speed measurements and theoretical considerations.

The experiment shows that the combustion process consists of the following essentially different stages. An induction 
period, during which the rod is heated up to a temperature at which an intensive metal oxidation begins at its upper end. This step is followed by a static combustion regime, during which liquid metal melted by the laser radiation slowly oxidizes, and a liquid "cap" grows on the upper rod end. Then, after a slow detachment of the liquid cap from the sample end, a dynamic combustion begins, which is characterized by a rapid metal consumption and much faster liquid motions. This is the main stage of the combustion process, insofar as most of the metal is burned within it. It turns out to be possible to give an analytical description of these stages which goes beyond mere order-of-magnitude estimates and in most cases provides a satisfactory quantitative account of the results of observations.

The paper is organized as follows. Section II describes the experimental setup. In Section III, we present and discuss our experimental results for pure iron and mild steel samples. The theoretical description is developed and compared to the experimental data in Section IV. Section V summarizes our results.

\section{EXPERIMENTAL SETUP AND PROCEDURE}

We studied combustion of $3.0 \mathrm{~mm}$-diameter iron and mild steel rods in an oxygen atmosphere at different pressures and different oxygen flow speeds. The experimental approach provides time-resolved surface temperature, ignition temperature, and high-speed imaging of the heating, ignition, and combustion stages of the metal specimens.

A schematic of the experimental setup used in this study is shown in Fig. 1. The setup includes a high-pressure combustion vessel, a high-speed camera, a mass flow controller, and a continuous fiber laser.

The high-pressure combustion vessel is a 13 1-capacity, Inconel cylindrical chamber designed to sustain static pressures up to 40 bars and equipped with five silica windows for optical access. Four windows provide visual access (perpendicular and at $45^{\circ}$, from above, to the cylinder axis), while the fifth, located at the top of the cylinder, lets the laser beam pass into the combustion chamber. The pres- sure inside the vessel is monitored by means of a pressure sensor.

The materials investigated are extra-pure iron (Goodfellow, FE007925, purity 99.99\%) and mild steel (S355J2, carbon mass fraction $0.2 \%$ ). The samples are cylindrical rods $15-35 \mathrm{~mm}$ in length and $3 \mathrm{~mm}$ in diameter. The oxidizing atmosphere is supplied by an oxygen gas stream (minimum purity $99.5 \%$ ) and is carried into the pressure vessel by a stainless-steel flexible tube before flowing out through the glass tube at a constant flow rate ranging from 0 to $45 \mathrm{~m}^{3} / \mathrm{h}$. The oxygen flow rate is externally adjusted by a manual valve and measured by a thermal mass flow meter.

Ignition is effected by heating up the sample's top surface by a disk laser (TRUMPF TruDisk 10002) operating at $1030 \mathrm{~nm}$. The laser beam is delivered through an optical fiber with a core diameter of $600 \mu \mathrm{m}$, providing a uniform intensity distribution. The beam is imaged onto the top of the rod by a set of three lenses $(200,650$, and $-1000 \mathrm{~mm})$. The circular beam spot size thus obtained is $3.1 \mathrm{~mm}$ in diameter, which ensures a homogeneous heating of the rod surface. Ignition was achieved by varying the laser intensity from 40 to $250 \mathrm{MW} \mathrm{m}^{-2}$ (corresponding to the laser power from $320 \mathrm{~W}$ to $2 \mathrm{~kW}$ ) and the pulse duration from $5 \mathrm{~ms}$ to $1 \mathrm{~s}$.

The sample burning was visualized using a high-speed camera (Ultima 1024 Photron), with the optical axis perpendicular to the sample axis (position 1), which may operate at a frame rate up to $4 \mathrm{kHz}$. Measurement of the top surface temperature of the rod during the ignition and combustion was achieved by recording the heating radiation emitted by the burning sample in the 800-950 $\mathrm{nm}$ wavelength range, with the same high-speed video camera (position 2, camera axis tilted $45^{\circ}$ with respect to the rod axis). Details of the pyrometer calibration are given in Ref. 23. The camera and the laser were triggered by the same signal, ensuring synchronous data acquisition. The timer is set so that the instant $t=0$ is the beginning of the laser pulse.

The experiment proceeds as follows. The top surface of each sample is first treated with a rough sand paper to ensure sufficient and repeatable absorptivity of the laser radiation. The sample is then housed inside the vessel, where it is fixed at

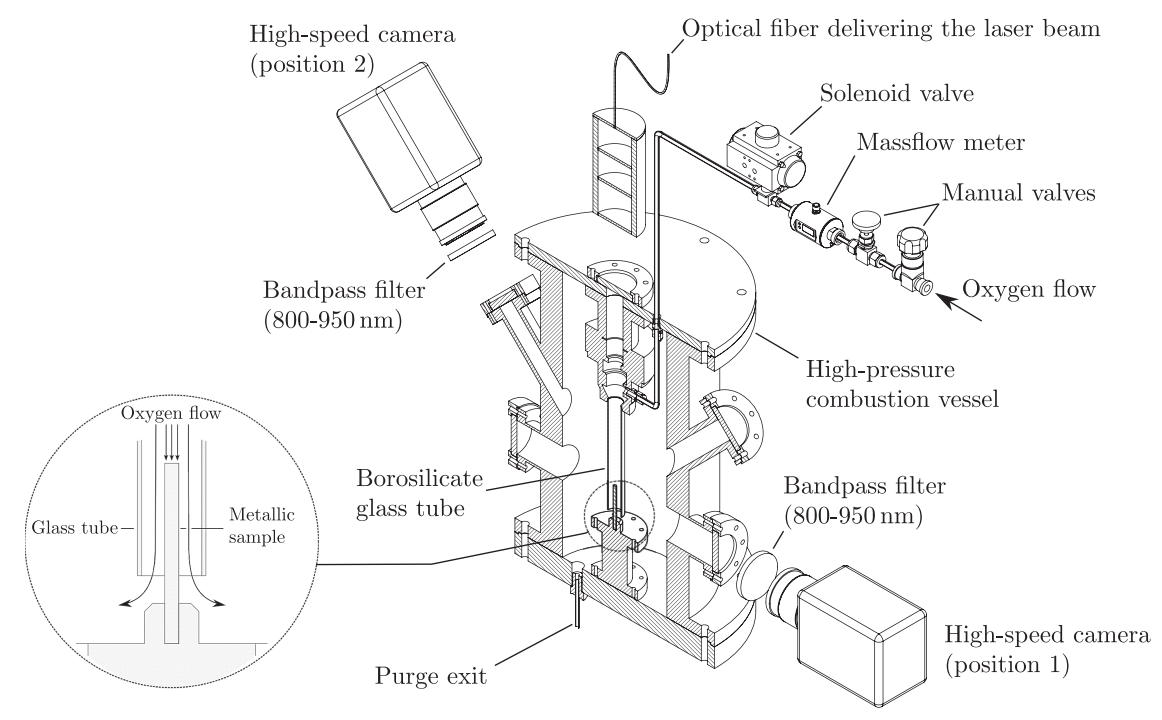

FIG. 1. Experimental setup. 
its bottom in a small chuck and partly placed inside a borosilicate glass tube (with an inner diameter of $16 \mathrm{~mm}$ ). Next, the chamber is purged and filled with oxygen at the desired pressure. After that, an extra oxygen is allowed to stream inside the charged chamber through a glass tube. The rate of the oxygen flow is adjusted by a manual valve so as to reach the desired value of the enclosure pressure. Once ready, the triggering signal is sent.

\section{EXPERIMENTAL RESULTS}

\section{A. Ignition and burning in a still oxygen atmosphere}

As a preliminary step, we studied metal combustion in the absence of oxygen flow under normal pressure.

The sequence of images in Fig. 2 shows the evolution of the top surface of a mild steel rod exposed to a $1 \mathrm{~kW}$ laser radiation. On the time interval $t \lesssim 10 \mathrm{~ms}$, the surface undergoes a slow solid-state oxidation process at temperatures below $840 \mathrm{~K}$. Above this temperature, the rate of oxidation significantly increases as is evidenced by the surface darkening due to a sharp increase of the surface absorptivity from 0.45 to 0.7. ${ }^{24}$ This behavior has been observed for iron and mild steel samples for all laser powers from 180 to $4000 \mathrm{~W}$, though the temperature at which it occurs increases with the laser power to about $1000 \mathrm{~K}$ at $4 \mathrm{~kW}$.

After this period of slow heating, during which the sample remains in a solid state, a much hotter luminous zone appears on the surface. The sharply increased luminosity clearly indicates the metal melting, which is also confirmed by the loss of granular structure on the surface (see Fig. 3(a) at $t=702 \mathrm{~ms}$ ). For a sufficiently low laser power, Fig. 3(a), this area first appears at the center and then extends over the entire surface. At higher laser powers, however, melting occurs uniformly over the surface due to the low surface temperature gradient, Fig. 3(b). No ignition was ever observed when the irradiation was interrupted before the onset of melting.

The melting of the top surface was tracked by the highspeed camera (in position 2) during the laser heating process in oxygen leading to ignition with various laser powers. Emissivity of the sample was assumed to be 0.75 when the temperature exceeds $840 \mathrm{~K}$, in accordance with the absorptivity measure- ments reported in Ref. 24. Temperature measurements were averaged over a spot of about $0.5 \mathrm{~mm}$ in diameter at the center of the top surface.

Figure 4 shows typical averaged temperature of an iron and mild steel rods during the last stage of heating by a $320 \mathrm{~W}$ laser. The curves consist of three clearly distinguished pieces. Before ignition, piece (A), the rate of the surface temperature increase is controlled by the laser: it is a resultant of the laser energy input and the energy loss due to the heat conduction into the sample bulk (in the present case this rate is approximately $5 \times 10^{3} \mathrm{~K} \mathrm{~s}^{-1}$ ). Piece (B) is characterized by a significantly larger slope: the rate sharply rises to about $140 \times 10^{3} \mathrm{~K} \mathrm{~s}^{-1}$. This means that a new mechanism of heat production comes into play, namely, the iron oxidation. Indeed, the rate changeover occurs at $1650 \mathrm{~K}$, which corresponds to the melting point of FeO. ${ }^{25}$ It is thus explained by an enhanced supply of oxygen to the fresh metal. In fact, regarding the species transport, liquid $\mathrm{FeO}$ is characterized by high ion mobilities and is thus more like a chemical electrolyte rather than a melted compound. In particular, the oxygen diffusion coefficient in liquid $\mathrm{FeO}\left(D \approx 4 \cdot 10^{-3} \mathrm{~cm}^{2} \mathrm{~s}^{-1}\right.$ at $\left.T=1900 \mathrm{~K}^{33}\right)$ is several orders of magnitude larger than that in solid $\mathrm{FeO}$ (which is about $\left(10^{-6}-10^{-5}\right) \mathrm{cm}^{2} \mathrm{~s}^{-1}$ at $T=1000 \mathrm{~K}$ to $\left.T=1500 \mathrm{~K}\right)$, a thin layer of which covers the metal and grows during the heating stage prior to ignition. Our experiments show that this layer retains its protective properties up to $1650 \mathrm{~K}$ for all laser powers in the range $180 \mathrm{~W}$ to $2 \mathrm{~kW}$. Furthermore, we observed a similar behavior of iron rods under elevated oxygen pressures (10 and 20 bars). This is as it should be according to the above explanation, for the melting point of $\mathrm{FeO}$ changes insignificantly with pressure. ${ }^{26}$

But shortly after the sharp rise, the heating rate drops down to approximately the same value as in (A), which happens at around $2300 \mathrm{~K}$. This means that above this temperature, on piece (C), the heat released by iron oxidation is spent almost completely on the iron melting. The iron melting point itself, $T_{m}=1811 \mathrm{~K}$, is not distinguished in any way on the plots of Fig. 4, because of the latent heat. The heat of iron fusion is $Q_{m}$ $=13.8 \mathrm{~kJ} \mathrm{~mol}^{-1}$, while its heat capacity $c_{p}=25.1 \mathrm{~J}(\mathrm{~mol} \mathrm{~K})^{-1}$, so that the iron melting fully establishes only at a temperature $T_{a}=T_{m}+Q_{m} / c_{p} \approx 2360 \mathrm{~K}$, and then begins to spread down the rod. As the result, a liquid drop grows at the upper rod

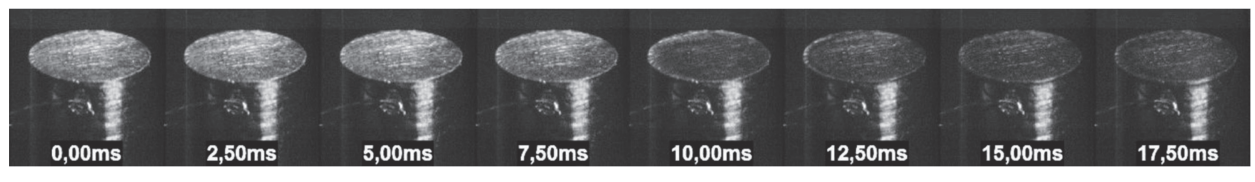

(a)

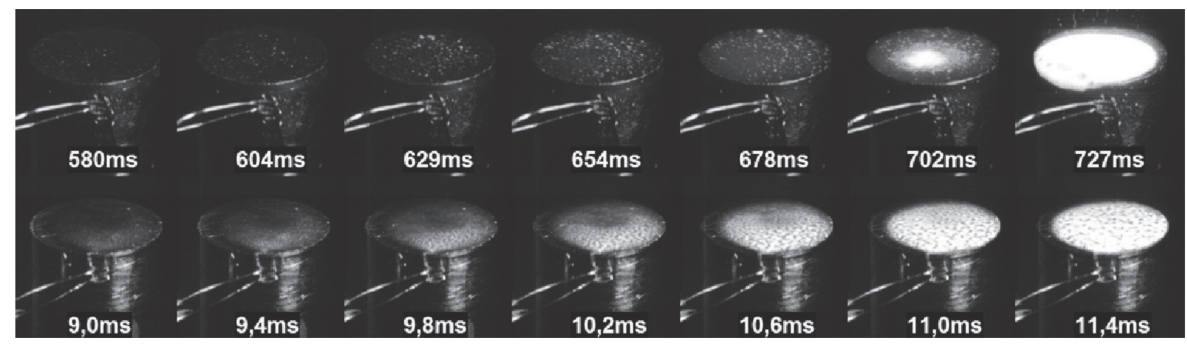

FIG. 2. Top surface of a mild steel rod during laser heating at $1 \mathrm{~kW}$ in still oxygen.
FIG. 3. Top surface of a mild steel rod during laser ignition: (a) $180 \mathrm{~W}$, (b) $1.5 \mathrm{~kW}$. 


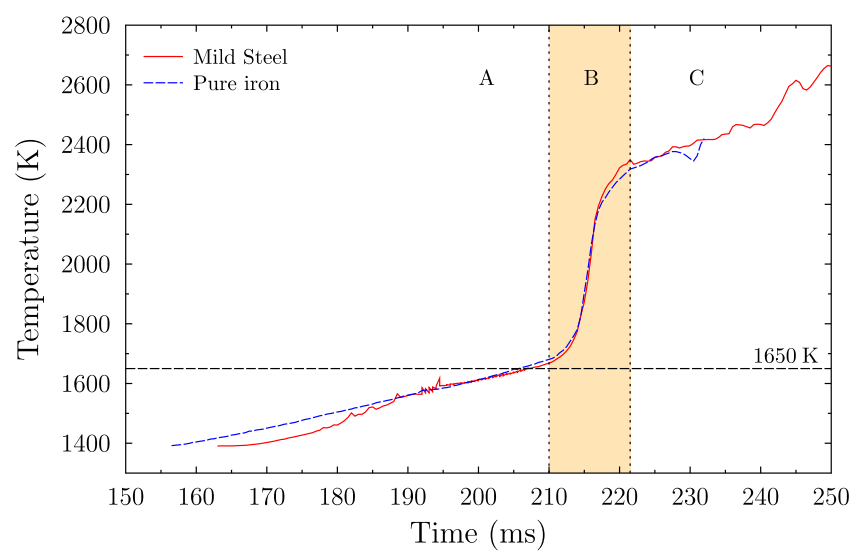

FIG. 4. The top surface temperature of iron and mild steel rods during laser irradiation at $320 \mathrm{~W}$ in oxygen.

end, while its apparent temperature (that is, temperature of the drop's top surface) continues to rise, though in a much less regular way, because of the more complicated heat transfer in the moving melt. It can reach values as high as $2900 \mathrm{~K}$, depending on the laser power and the exposure time.

A close coincidence of the two curves in Fig. 4 demonstrates that the heating and ignition of iron and mild steel rods proceed essentially in the same way not only qualitatively but also quantitatively. This implies, in particular, that the possible effect of $\mathrm{CO}$ formation in the case of mild steel is negligible. We will have more to say regarding this point later on in Sec. IV C 1.

Once the laser ceases, only the heat from the oxidation reaction is transferred to the rod. If it is not enough to melt the fresh metal, the burning liquid gradually cools down and eventually extinguishes. Otherwise, the combustion enters in a self-sustained regime when the fresh molten metal is delivered into the burning drop until it detaches from the rod, leaving a small amount of molten material on its top. In this case, the liquid temperature decreases and settles at approximately 2400 $\mathrm{K}$. The cycle of the drop growth and its detachment then repeats itself until the sample is consumed. Time-resolved images of both situations are shown in Fig. 5, for pure iron and the laser power of $640 \mathrm{~W}$. A comprehensive description of this process using the phase diagram of the iron-oxygen system is given in Ref. 27.

\section{B. Burning in an oxygen flow. Normal pressure}

We now turn to the combustion of metal rods subject to an oxygen flow. Our aim is to study the effects of the oxygen flow speed and its pressure.

Figures 6-8 are sequences of images of mild steel rods burning in oxygen flowing at speeds from $v_{0}=1.7 \mathrm{~m} \mathrm{~s}^{-1}$ to $v_{0}=60 \mathrm{~m} \mathrm{~s}^{-1}$ and various laser powers. In all tests performed, the combustion process ultimately evolved into one of the two different self-sustained regimes. At the low flow speeds $v_{0} \lesssim$ $1.7 \mathrm{~m} \mathrm{~s}^{-1}$, Figs. 6(a) and 6(b), the rod burning is quite similar to that observed in the experiments with no oxygen flow, that is, consumption of the rod proceeds through the cycle of growth and detachment of a liquid cap. The detached molten material was often observed to precess around the burning rod as it was running down along it. A different behavior was observed for $v_{0} \gtrsim 2 \mathrm{~m} \mathrm{~s}^{-1}$. Figures 6(c), 6(d), 7, and 8 show that in this case, the liquid continues to flow down after the drop fall, which leads to a gradual erosion of the rod on one of its sides with an increasingly steep slope. It never progresses in a symmetrical way with respect to the rod axis.

The measured temperature and position of the upper end of a mild steel rod are plotted against time in Fig. 9. The upper end position, $x(t)$, is defined hereinafter as the vertical distance between an initially horizontal top surface of the rod before ignition and its highest point at instant $t$. In all cases presented in this figure, the laser is switched off once the sample was ignited so that the subsequent rod burning is self-sustained. We observe that

- The onset of propagation (indicated in the graphs by "fall of the liquid") always corresponds to a considerable decrease of the surface temperature which varies significantly with the oxygen flow speed: whereas it is in the range $2250-2500 \mathrm{~K}$ for $v_{0}=10 \mathrm{~m} \mathrm{~s}^{-1}$, it drops down to $1950-2100 \mathrm{~K}$ for $v_{0}=60 \mathrm{~m} \mathrm{~s}^{-1} .^{28}$

- The rate of rod regression grows rapidly with the oxygen flow speed.

- The temperature and position curves for $v_{0} \geqslant 10 \mathrm{~m} \mathrm{~s}^{-1}$ show a good collapse upon matching the moments corresponding to the onset of dynamic combustion.

The last observation means that for sufficiently large oxygen flow speed, the properties of dynamic combustion are (a)

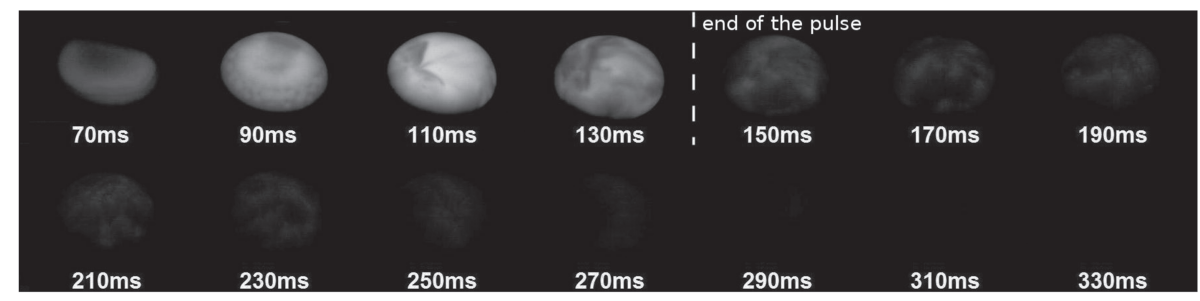

(b)

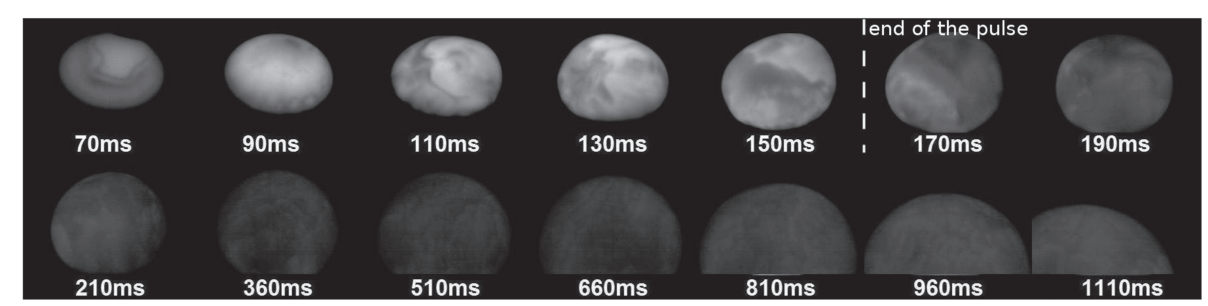

FIG. 5. Infrared images (800-950 nm) of a pure iron rod while burning in the static combustion regime $(640 \mathrm{~W})$ for pulse durations of (a) $130 \mathrm{~ms}$ leading to extinction; (b) $150 \mathrm{~ms}$ leading to self-sustained combustion and then to propagation (modified contrast). 


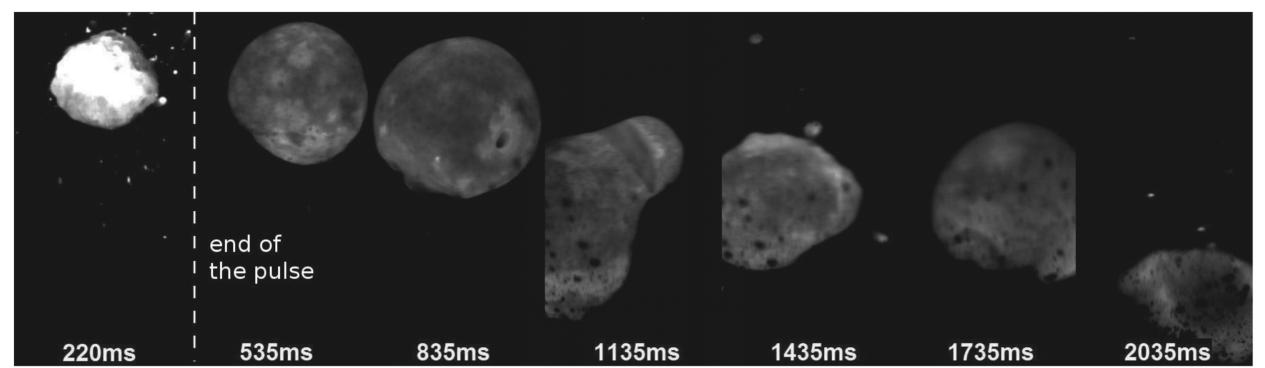

(a) Oxygen flow speed : $1.7 \mathrm{~m} \cdot \mathrm{s}^{-1}$, laser power : $640 \mathrm{~W}$, laser pulse duration : $235 \mathrm{~ms}$.

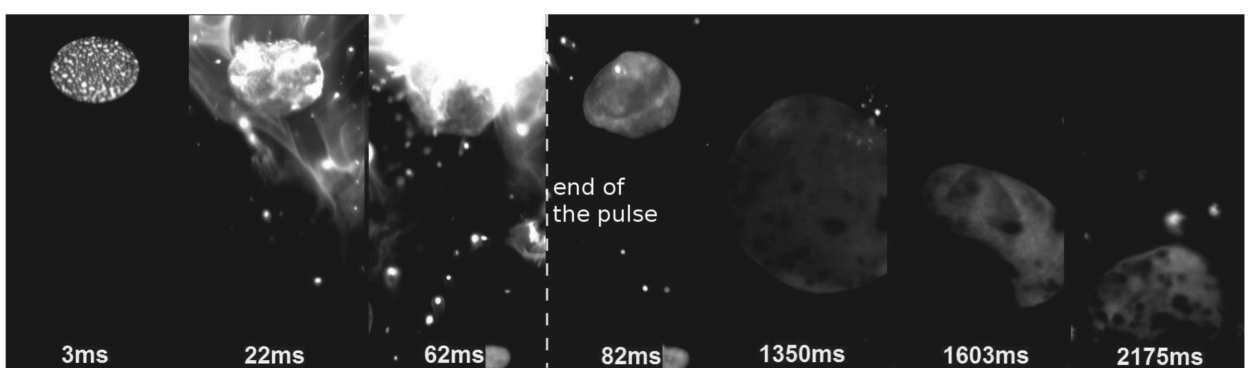

(b) Oxygen flow speed : $1.7 \mathrm{~m} \cdot \mathrm{s}^{-1}$, laser power : $4 \mathrm{~kW}$, laser pulse duration : $65 \mathrm{~ms}$.

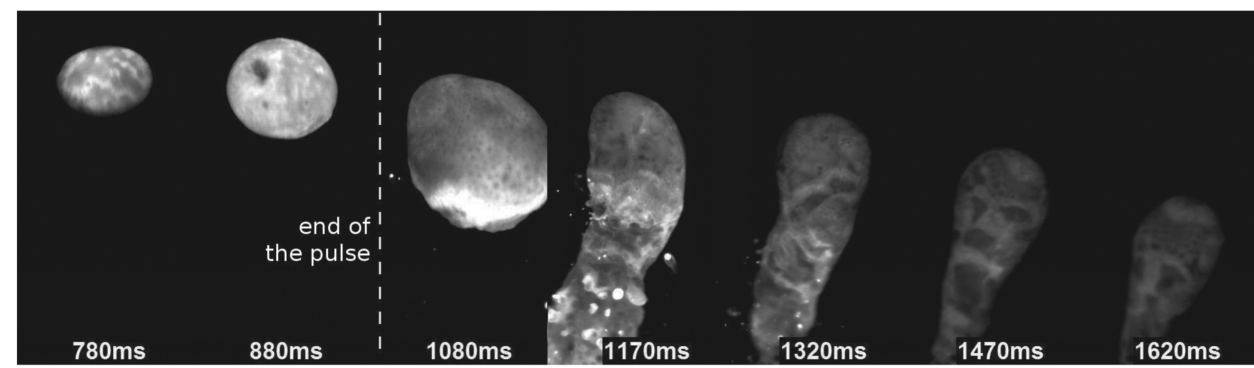

(c) Oxygen flow speed : $10 \mathrm{~m} \cdot \mathrm{s}^{-1}$, laser power : $180 \mathrm{~W}$, laser pulse duration : $950 \mathrm{~ms}$.

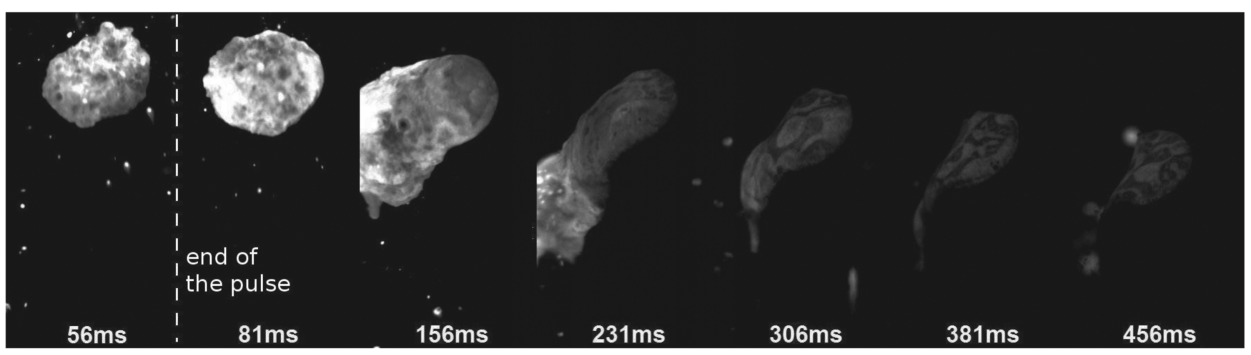

(d) Oxygen flow speed : $10 \mathrm{~m} \cdot \mathrm{s}^{-1}$, laser power : $2 \mathrm{~kW}$, laser pulse duration : $60 \mathrm{~ms}$.
FIG. 6. Infrared images $(800-950 \mathrm{~nm})$ for the propagation of combustion on mild steel rods for different oxygen flow speeds and different laser powers. The laser input energies are at the propagation thresholds. independent of the preceding rod evolution, in particular, of the laser power and other specifics of ignition. The main goal of the subsequent theoretical development is to describe analytically these properties, in particular, to account for the observed increase of the metal consumption rate with the oxygen flow speed. Experimental results on the rod combustion under elevated pressures will be presented afterwards.

\section{ANALYTICAL DESCRIPTION OF THE ROD COMBUSTION}

The series of side-view images in Fig. 10 represents a typical history of the rod evolution showing its main stages: an induction period preceding the static combustion, an initial development of which is seen on the second frame, and which ends at $t=438 \mathrm{~ms}$ with the fall of the liquid cap; it is followed by the rapid dynamic combustion characterized by a large slope of the interface between solid metal and thin liquid flow. In this natural order, these stages are described analytically in Secs. IV A-IV C, respectively.

\section{A. Induction stage}

According to the above-described experimental conditions, we consider an iron or steel cylindrical sample placed vertically and assume that its length, $L$, is much larger than the diameter $d$ of its circular cross section. Let the upper end of the sample be laser-irradiated for a time $t_{e}$, the power $I$ of the electromagnetic radiation being uniformly distributed 


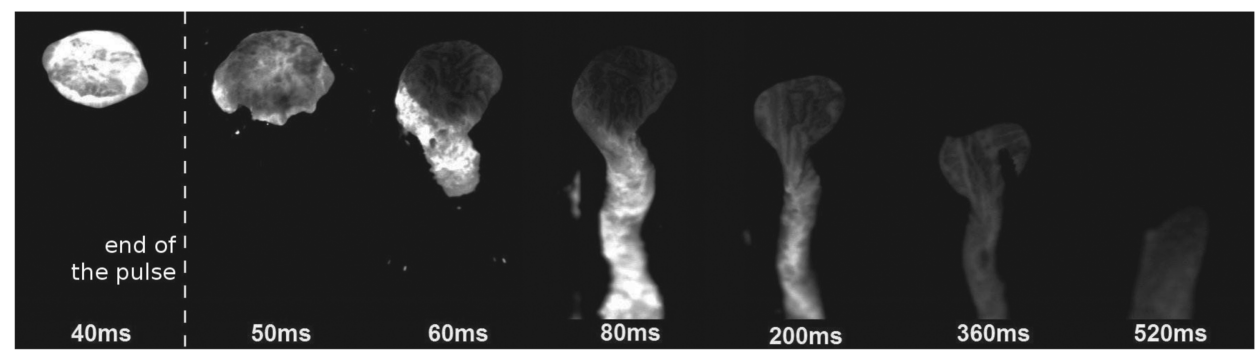

(a) Oxygen flow speed : $30 \mathrm{~m} \cdot \mathrm{s}^{-1}$, laser power : $1 \mathrm{~kW}$, laser pulse duration : $50 \mathrm{~ms}$.

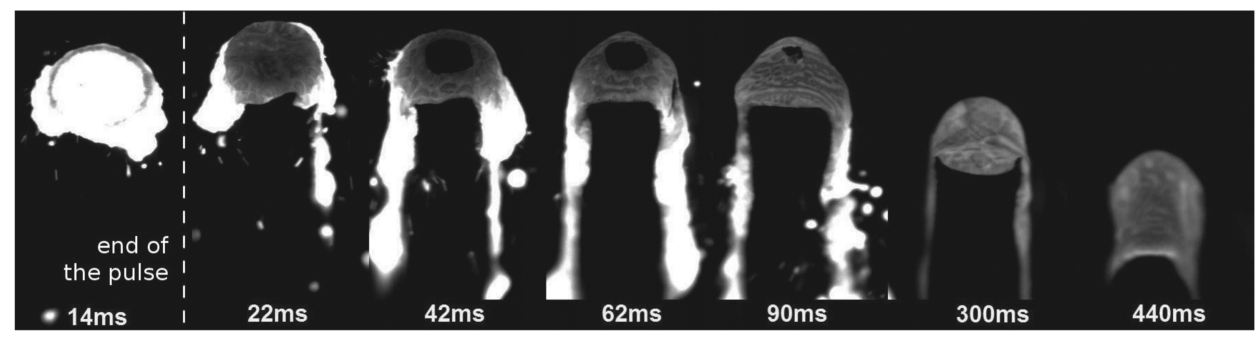

(b) Oxygen flow speed : $30 \mathrm{~m} \cdot \mathrm{s}^{-1}$, laser power : $3 \mathrm{~kW}$, laser pulse duration : $15 \mathrm{~ms}$.

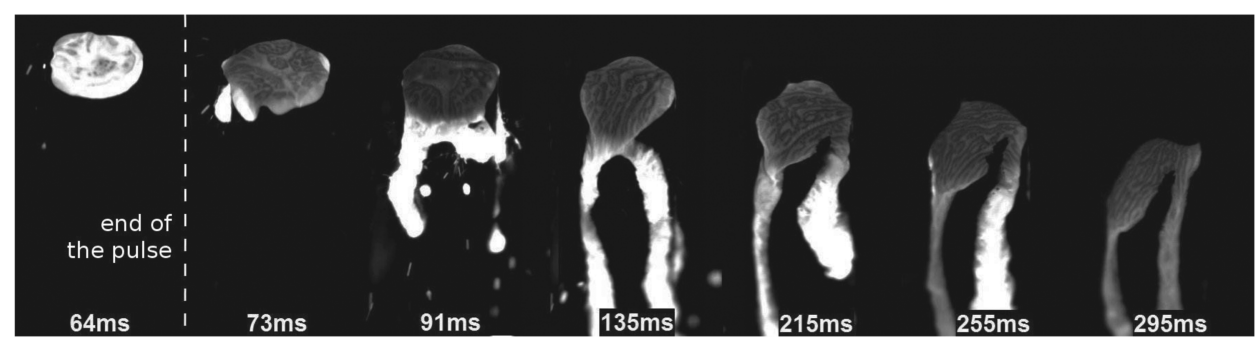

FIG. 7. Same as in Fig. 6.

(c) Oxygen flow speed : $40 \mathrm{~m} \cdot \mathrm{s}^{-1}$, laser power : $640 \mathrm{~W}$, laser pulse duration : $72 \mathrm{~ms}$.

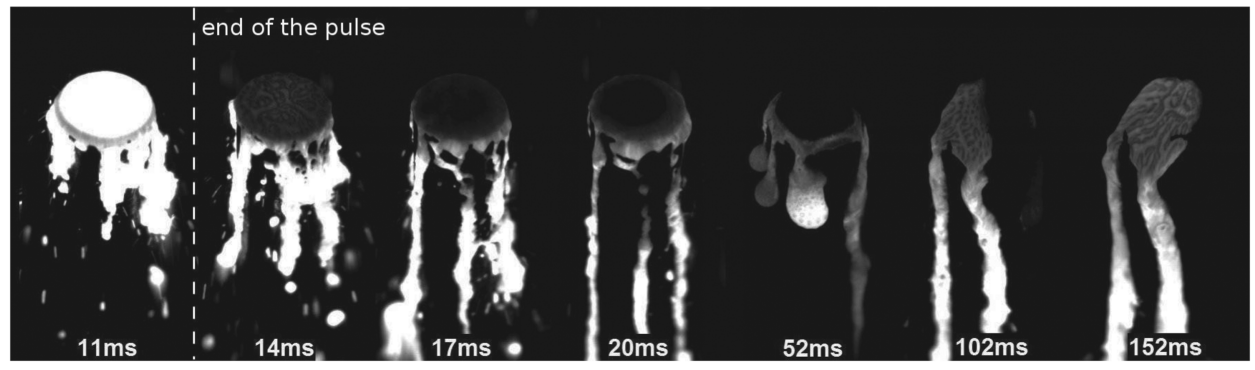

(d) Oxygen flow speed : $50 \mathrm{~m} \cdot \mathrm{s}^{-1}$, laser power : $4 \mathrm{~kW}$, laser pulse duration : $12 \mathrm{~ms}$.

across the top surface and constant during the exposure time, Fig. 10. The process of sample heating can be considered as one-dimensional, because the top surface is perpendicular to the sample axis, the radiation heat loss is easily seen to be negligible, and the sample is initially homogeneous (in particular, its initial temperature $T_{0}$ is uniform). Therefore, it is described by the following equation:

$$
\frac{\partial T}{\partial t}=\chi \frac{\partial^{2} T}{\partial x^{2}}+\alpha \delta(x), \quad \alpha=\frac{2 I}{\rho c_{p} s^{\prime}},
$$

where $x$ is the axial co-ordinate, $\chi, \rho$, and $c_{p}$ are the metal thermal diffusivity, density, and specific heat, respectively, $s=\pi d^{2} / 4$, and Dirac's function describes the heat release due to the irradiation of the surface $x=0$. Assuming $\chi, \rho, c_{p}$ independent of temperature, the solution of this equation, satisfying given initial ( $T=T_{0}$ at the initial instant $t=0$ ) and boundary conditions (absence of heat losses at the sample surface), reads

$$
T(x, t)=T_{0}-\frac{\alpha|x|}{2 \chi}+\frac{\alpha}{4 \sqrt{\pi \chi^{3} t}} \int_{-\infty}^{+\infty} d x^{\prime}\left|x^{\prime}\right| \exp \left\{-\frac{\left(x-x^{\prime}\right)^{2}}{4 \chi t}\right\} .
$$

The integral over $x^{\prime}$ is to be taken here over a domain symmetric with respect to the origin to guarantee the vanishing of the heat flux at the upper rod end, whereas extending this domain over the whole infinite line means the neglect of the lower end effects.

As intensive metal oxidation (i.e., ignition) begins once the protective layer of the metal oxide is melted, the ignition time, $t_{i}$, is equal to the exposure time needed to reach the FeO melting temperature, $T_{m}^{\prime}=1650 \mathrm{~K}$, at the surface $x=0$. 


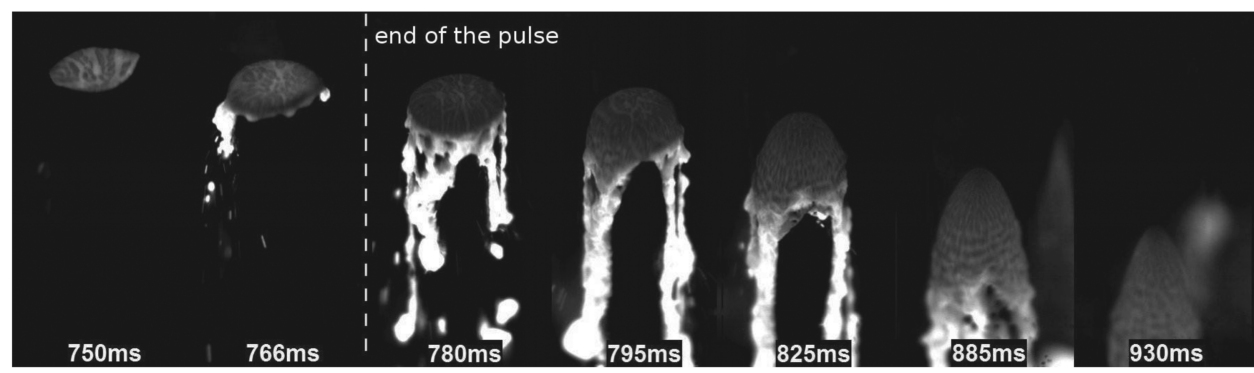

(a) Oxygen flow speed : $30 \mathrm{~m} \cdot \mathrm{s}^{-1}$, laser power : $180 \mathrm{~W}$, laser pulse duration : $780 \mathrm{~ms}$.

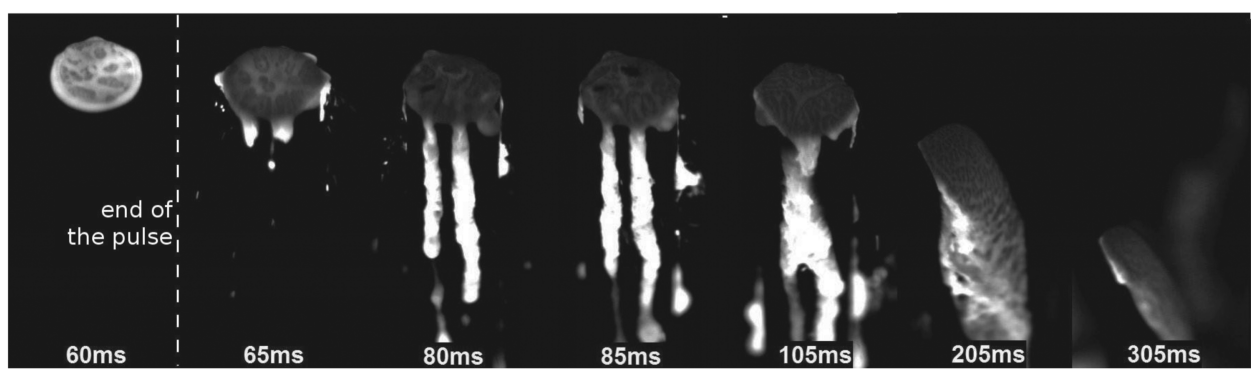

FIG. 8. Same as in Fig. 6.

(b) Oxygen flow speed : $60 \mathrm{~m} \cdot \mathrm{s}^{-1}$, laser power : $640 \mathrm{~W}$, laser pulse duration : $65 \mathrm{~ms}$.

Therefore, we find from Eq. (2)

$$
T_{m}^{\prime}-T_{0}=\frac{\alpha}{4 \sqrt{\pi \chi^{3} t_{i}}} \int_{-\infty}^{+\infty} d x^{\prime}\left|x^{\prime}\right| \exp \left\{-\frac{x^{\prime 2}}{4 \chi t_{i}}\right\}=\alpha \sqrt{\frac{t_{i}}{\pi \chi}}
$$

which after substitution of the expression for $\alpha$ yields the induction time

$$
t_{i}=\frac{\pi \chi}{4}\left[\frac{\rho c_{p}\left(T_{m}^{\prime}-T_{0}\right) s}{I}\right]^{2}
$$

Since the thermal diffusivity of metals noticeably depends on temperature, this formula can be used to compare $t_{i}$ for different $I$ with $\chi$ set to some mean value (dependent on $T_{m}^{\prime}$ ) specific to the given material.

For the purpose of experimental verification, it is convenient to rewrite this formula by noting that it implies a linear relation between the inverse laser power and the total energy, $E=I t_{i}$, transferred to the sample during the induction period, namely,

$$
E=\frac{\beta}{I}, \quad \beta \equiv \frac{\pi \chi}{4}\left[\rho c_{p}\left(T_{m}^{\prime}-T_{0}\right) s\right]^{2}
$$

$E$ versus $I^{-1}$ for an iron rod with $d=3 \mathrm{~mm}$ is plotted in Fig. 11. For comparison, data for a stainless steel (316L, 17\% chromium content by mass) rod of the same dimensions are also shown. It is seen that stainless steel requires about 1.5 more energy than iron to ignite. This is because of the much higher temperature required to melt the protective oxide layer $\left(\mathrm{Cr}_{2} \mathrm{O}_{3}, T_{m}^{\prime}=2708 \mathrm{~K}\right)$ [though the thermal diffusivity of stainless steel is several times lower than that of iron at room temperature, the difference is much smaller at higher temperatures relevant to ignition, and the effect of the factor $\left(T_{m}^{\prime}-T_{0}\right)^{2}$ in Eq. (4) overrides that of the $\chi$-factor].

\section{B. Static combustion}

Subsequent to ignition is the stage of static combustion. As we have seen, during this stage liquid metal melted by the laser radiation is slowly oxidized, the energy released in the chemical reaction being spent primarily on further reheating of the liquid cap growing on the upper rod end. The heat outflow from this region to the solid part is weak despite the temperature rise, because the cap thickness is comparatively large (of the order of the rod diameter), so that the temperature gradient is relatively small. The shape of the cap and its kinematics are controlled by the surface tension and gravity. The latter affects the liquid in two ways: one is the usual bulk force acting on each liquid element and the other is the baroclinic effect-gravity-induced vorticity generation. The value of the liquid velocity curl, $\omega$, generated on crossing the solid-liquid interface is ${ }^{29}$

$$
\omega_{g}=\frac{\theta-1}{\theta} \frac{g \sin \varphi}{u_{n}} .
$$

where $g$ is the gravity acceleration, $u_{n}$ the normal speed of the solid-liquid interface relative to the cold solid, $\varphi$ its local inclination angle with respect to the horizontal, and

$$
\theta=\frac{\rho}{\rho_{l}}
$$

$\rho\left(\rho_{l}\right)$ being the solid (liquid) density. Vorticity of the liquid elements will be further amplified as they travel from the solidliquid interface into the bulk, by essentially the same baroclinic mechanism, due to the temperature increase (hence, density decrease) away from the interface.

If the solid-liquid interface remains horizontal $(\varphi=0)$, then $\omega_{g}=0$ and nothing prevents the cap from flowing down the rod when the surface tension fails to hold the cap, that is, when its size exceeds the rod diameter by more than the capillary length. Because of the surface tension, the liquid does not fall as one layer spreading uniformly around the rod but 


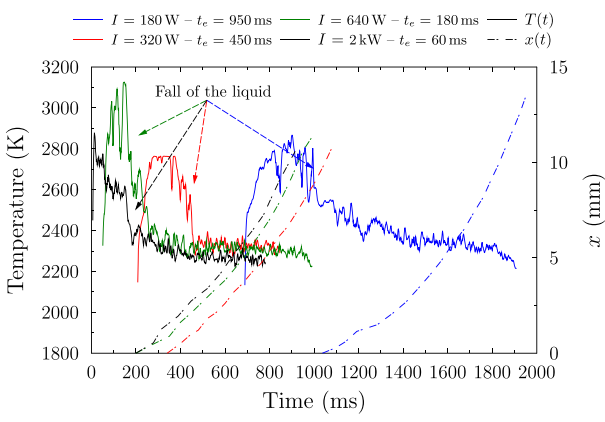

(a) Oxygen flow speed : $10 \mathrm{~m} \cdot \mathrm{s}^{-1}$

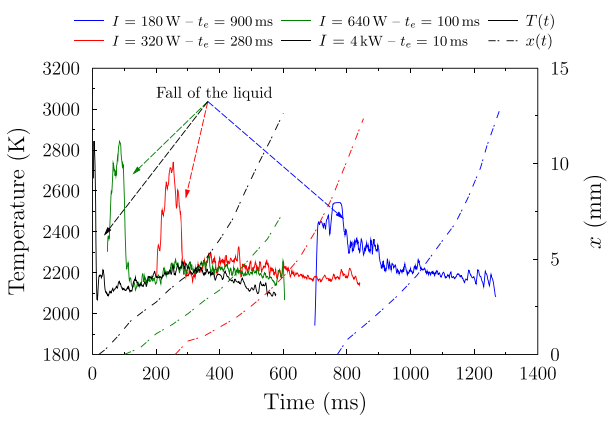

(c) Oxygen flow speed : $30 \mathrm{~m} \cdot \mathrm{s}^{-1}$

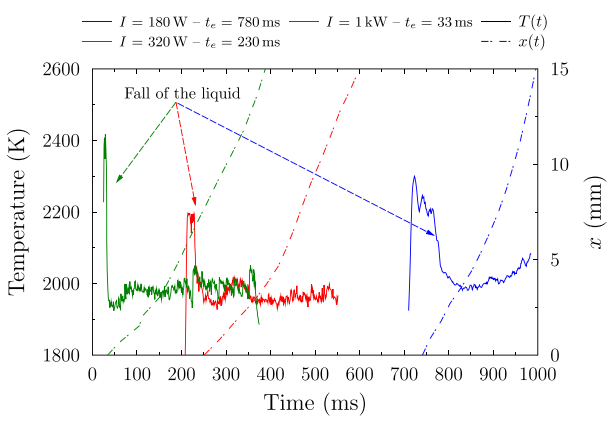

(e) Oxygen flow speed : $60 \mathrm{~m} \cdot \mathrm{s}^{-1}$

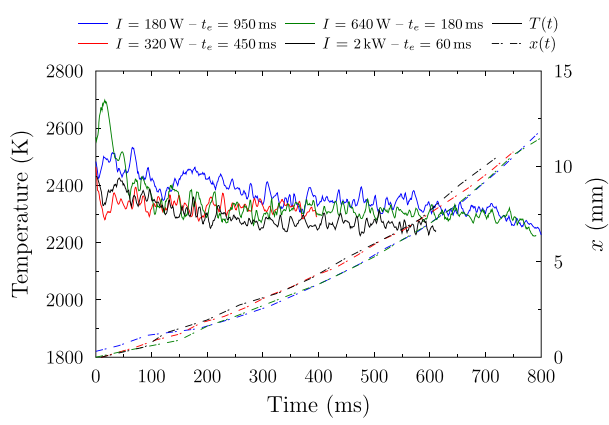

(b) Oxygen flow speed : $10 \mathrm{~m} \cdot \mathrm{s}^{-1}$, shifted time

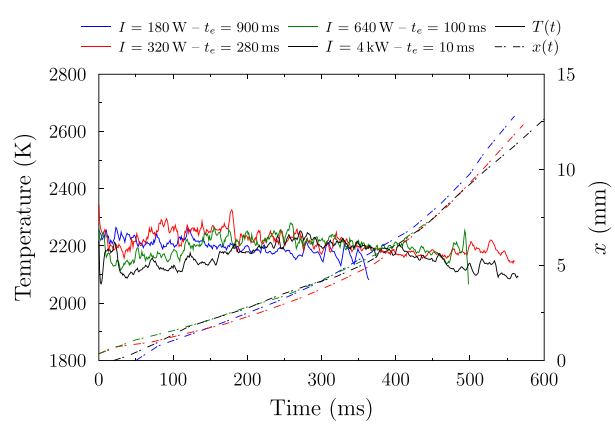

(d) Oxygen flow speed : $30 \mathrm{~m} \cdot \mathrm{s}^{-1}$, shifted time

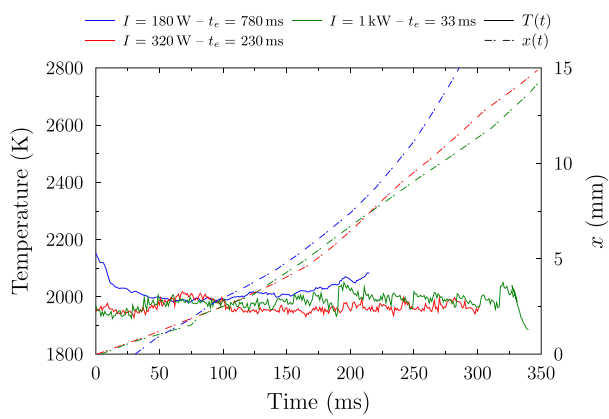

(f) Oxygen flow speed : $60 \mathrm{~m} \cdot \mathrm{s}^{-1}$, shifted time

FIG. 9. Temperature and position of the rod's top surface against time for different oxygen flow speeds. The curves in (b), (d), and (f) are the same as in (a), (c), and (e) but shifted in time so that $t=0$ corresponds to the fall of the liquid.

rather tends to gather in one drop that falls on one side of the rod. This brings uncontrollable asymmetry into the process.

Things get more complicated when the solid-liquid interface becomes inclined during the liquid cap evolution (again, azimuth of the inclination with respect to the rod axis is uncontrollable). The point is that the local rotation of the liquid elements, generated by the baroclinic effect, tends to roll them up the inclined interface (that is, the rotation is counterclockwise on the sketch shown in Fig. 12 so that its angular velocity, equal to $\omega / 2$, is positive). According to the Thomson theorem, velocity circulation is conserved in an ideal constant-density flow in the presence of a homogeneous gravity field, which in the simplified two-dimensional representation of Fig. 12 corresponds to the conservation of $\omega$. As was just mentioned, the liquid density is not constant, but the density gradient is such that $\omega$ is further increased as the liquid elements travel away from the solid-liquid interface. On the other hand, a liquid flowing down along the rod would have $\omega$ of the opposite sign. In a truly two-dimensional flow, therefore, the liquid cap would have to "wait" until this vorticity is decayed by the viscous friction, before it starts to move along an inclined surface. In the actual three-dimensional situation, however, vorticity may reverse its sign without assistance of the viscous forces, via fragmentation of the flow into vertical cells leaning over in the transverse direction, which results in a vorticity flip-over. In any case, the cap motion is comparatively slow as it is driven by the slow ordinary oxygen diffusion and heat conduction in a laminar flow. In view of this, it can be naturally called a slow onset of dynamic combustion.

The cap detachment becomes more rapid in the presence of a downward oxygen flow, because of the viscous drag exerted on it by the gas. Also, if the flow speed is high enough to turbulize the liquid, the oxygen consumption rate greatly increases because the oxygen transfer through the liquid flow is enhanced by the turbulent pulsations. We then have a fast onset of dynamic combustion. A quantitative criterion 


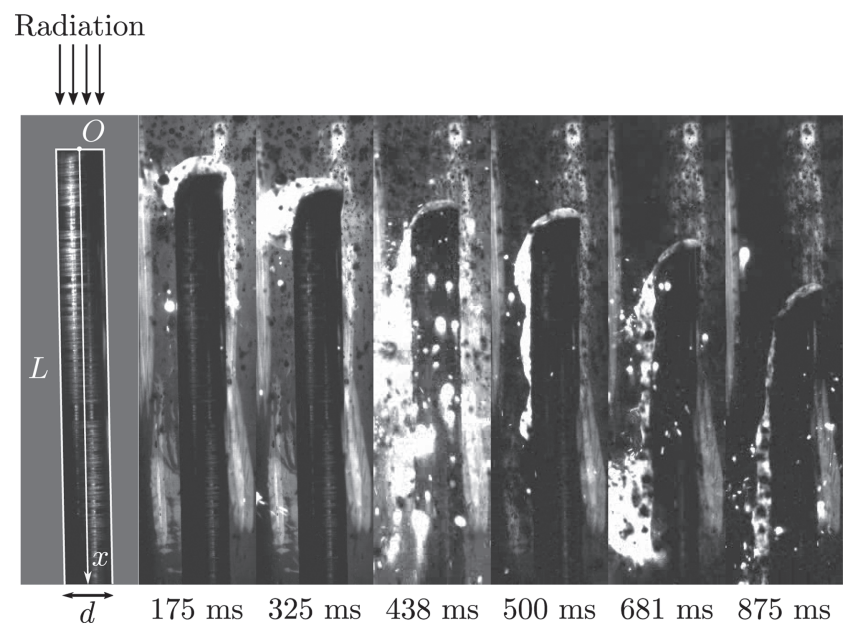

FIG. 10. Regression of a carbon steel rod in an oxygen flow of $10 \mathrm{~m} \mathrm{~s}^{-1}$ speed.

distinguishing the two cases is the minimal shear stress of the oxygen flow required to compensate the baroclinic vorticity, $\tau=\eta \omega_{g}$ ( $\eta$-dynamic viscosity of the liquid). Writing $\tau=\rho_{0} v_{*}^{2}$, where $\rho_{0}$ is the oxygen density, the corresponding friction velocity $v_{*}$ can be estimated using the law of the wall discussed in Sec. IV C.

\section{The theory of dynamic combustion}

The experimental evidence presented in Sec. III B is that in a sufficiently fast oxygen flow, the slow detachment of a liquid cap from the upper rod end gives way to an entirely different combustion regime, the dynamic combustion, which is characterized by a rapid consumption of the metal. The slope $\varphi$ of the solid-liquid interface is now large, the liquid mixture of melted metal and oxide streaming down along it as is illustrated by the right half of Fig. 10 and schematized in Fig. 13. The vertical propagation speed of the interface in the laboratory reference frame, $u=d x / d t$, is typically $1 \mathrm{~cm} \mathrm{~s}^{-1}$

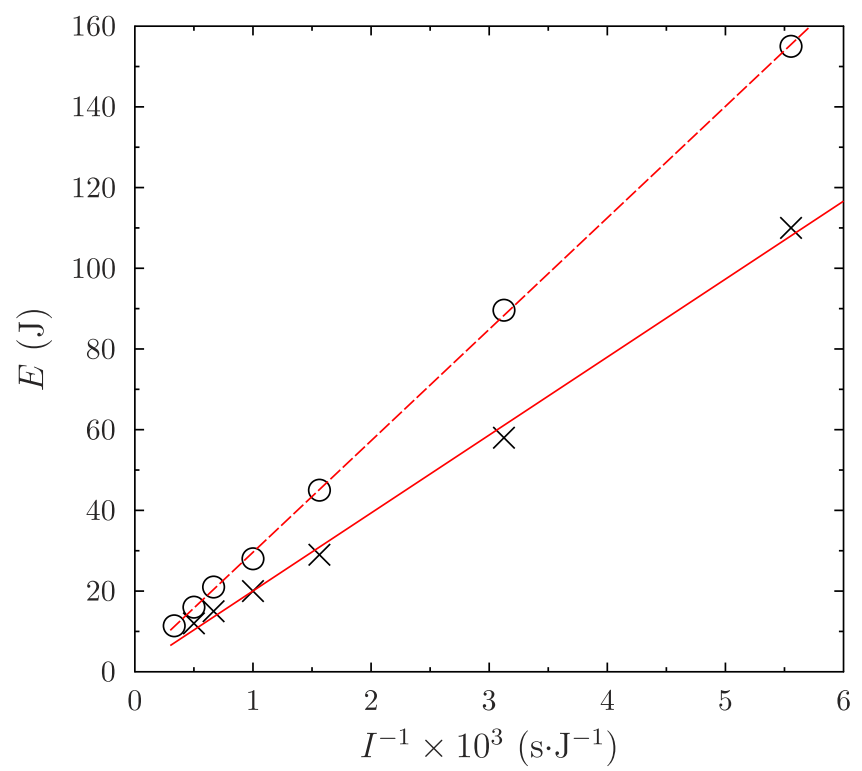

FIG. 11. Total energy required to ignite iron (solid line, crosses) and stainless steel (broken line, open circles) rods of $3 \mathrm{~mm}$ diameter as a function of inverse laser power. Lines represent best linear fits, marks - experimental data.

\section{Oxygen flow}

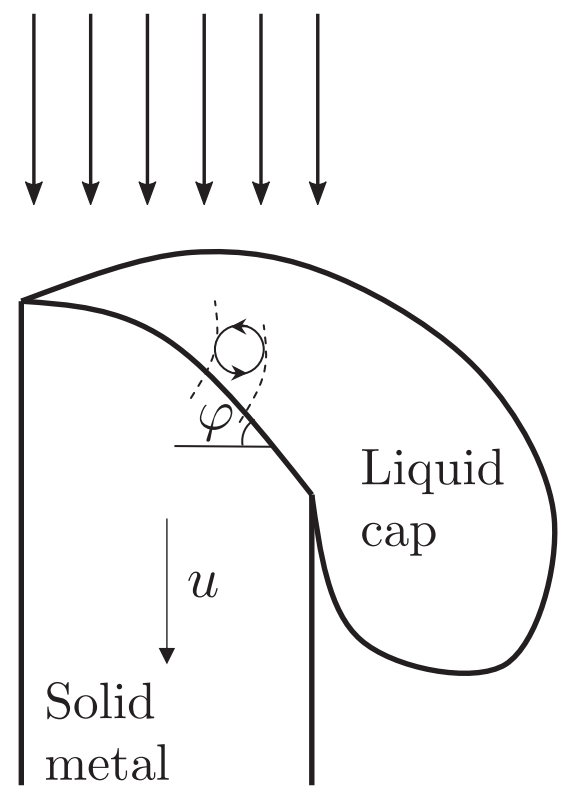

FIG. 12. Flow structure in the liquid cap on inclined interface. Shown are two neighboring stream lines right after they crossed the interface (broken lines) and a rotating fluid element.

to $10 \mathrm{~cm} \mathrm{~s}^{-1}$, but the liquid flow velocity, $v$, is significantly higher - usually by a factor of 10 to $10^{2}$, depending on the oxygen flow speed and pressure. By this reason, the liquid layer thickness, $h$, is small compared to the interface length, being normally less than $1 \mathrm{~mm}$. The quantities $u$ and $\varphi$ can be used to find the normal propagation speed of the solid-liquid interface relative to the solid according to $u_{n}=u \cos \varphi$, which is the primary aim of the subsequent consideration.

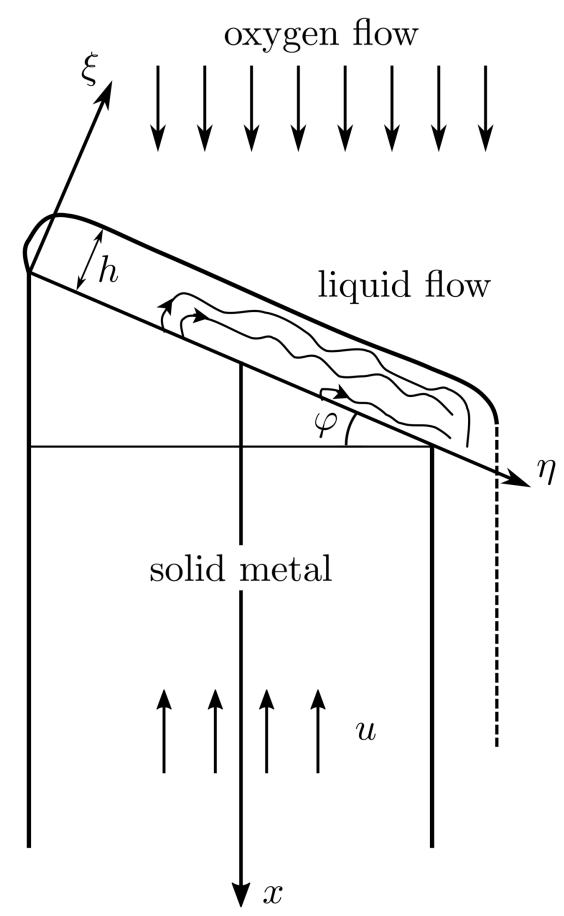

FIG. 13. Schematics of the upper rod end in the regime of dynamic combustion. 
As the described change of the burning regime is caused by the oxygen flow, it is its interaction with the liquid metal-oxide that drives the combustion process. The main characteristic of this interaction is the friction velocity $v_{*}$ that determines the shear stress exerted on the liquid by the flow and which can be estimated using the law of the wall

$$
v_{0} \approx 2.5 v_{*} \ln \frac{v_{*}^{2} d}{v_{0} v_{0}},
$$

where $v_{0}$ is the kinematic viscosity of the oxygen flow. The argument of the logarithm in this formula is the ratio of the boundary layer thickness and the viscous layer thickness, estimated as $v_{*} d / v_{0}$ and $v_{0} / v_{*}$, respectively. As these are only rough estimates, accuracy of this asymptotic formula is only logarithmic, that is, it requires the logarithm be sufficiently large. [It is known that the mean velocity profile of the boundary layer flows at not too large Reynolds numbers is often better approximated by a power-rather than the logarithmic law. ${ }^{30}$ However, a few-percent difference between the two approximations is well within the accuracy of the subsequent applications of Eq. (6).]

The following observation is of major importance for the analytical description of the dynamic stage. The thermal diffusivity of solid iron or steel is about $0.1 \mathrm{~cm}^{2} \mathrm{~s}^{-1}$ so that for a sample of several centimeters in length, the dimensionless ratio $u L / \chi \gg 1$. Substituting $L=u \Delta t$, where $\Delta t$ is the combustion time, we conclude that the dynamic stage is characterized by

$$
\frac{u^{2} \Delta t}{\chi} \gg 1 \text {. }
$$

This condition means that during most part of the combustion process the heat transfer can be treated as quasi-steady in the rest frame of the solid-liquid interface. Indeed, the heat transfer in the bulk of the sample obeys

$$
\frac{\partial T}{\partial t}+(\boldsymbol{u} \boldsymbol{\nabla}) T=\chi \Delta T .
$$

If the first term on the left of this equation was larger than the second, during a time of the order of $\Delta t$ (which is the only characteristic time in the rest frame of the solid-liquid interface held at a constant temperature $T_{m}$ ), the temperature would be appreciably raised at distances $\sim \sqrt{\chi \Delta t}$ from the upper rod end. But then the ratio of the first term on the left of Eq. (8) to the second would be $\sqrt{\chi / u^{2} \Delta t} \ll 1$, contrary to the assumption. Therefore, under condition (7) $\partial T / \partial t$ can be omitted, and the characteristic thermal length along the rod is actually $\chi / u$, as found by comparing the second term with the right-hand side of Eq. (8).

\section{Thermal structure of the liquid layer}

The images in Figs. 6-8 clearly demonstrate the presence of rapidly changing irregular structures on the liquid surface, and the more detailed video records show that the liquid moves chaotically as it flows along the solid-liquid interface, its velocity fluctuating in the spanwise as well as the streamwise directions. This apparent irregularity suggests that the liquid dynamics is turbulent, which is further confirmed by estimating the flow Reynolds number. According to the general recipe, this number can be calculated as $\operatorname{Re}=\Delta v \Delta l / v$, where $\Delta v$ is the change of the liquid velocity along a characteristic distance $\Delta l$, and $v$ is its kinematic viscosity. In the frame of reference attached to the solid-liquid interface, the velocity component parallel to the interface changes from $(-u \sin \varphi)$ to about $10-10^{2}$ times $u$ at the distance of order $d / \cos \varphi$, so that the product $\Delta v \Delta l \approx\left(10-10^{2}\right) u d$. On the other hand, kinematic viscosity of the liquid iron is rather small, $v \approx 5 \times 10^{-3} \mathrm{~cm}^{2}$ $\mathrm{s}^{-131}$ (viscosity of the iron oxide at the temperatures of interest is about the same value ${ }^{32}$ ). Therefore, Reynolds number of the liquid flow is large

$$
\operatorname{Re} \approx 10^{3}-10^{4}
$$

indicating turbulent conditions indeed. It can be added in this connection that the flow under consideration is inherently unstable also due to the vorticity effects discussed in Sec. IV B, which evidently provide the triggering and amplification of the turbulence.

An immediate important consequence of the above observation is that the oxygen transport in the liquid is the turbulent diffusion. Since the oxygen flow is also turbulent, the oxygen entrainment into the liquid proceeds primarily through the turbulent mixing at the liquid-oxygen interface. Since the turbulent transport delivers oxygen into the bulk of the liquid, it is far more efficient than the ordinary diffusion which under the present conditions affects only a thin surface layer because of the smallness of the oxygen diffusion coefficient $\left(D \approx 4 \cdot 10^{-3}\right.$ $\mathrm{cm}^{2} \mathrm{~s}^{-1}$ at $T=1900 \mathrm{~K}^{33}$ ). This mechanism is sketched in Fig. 14. Turbulent motions at the liquid-oxygen interface enrich the liquid in the near-interface region with oxygen, which is shown in the figure as a brightening of the liquid breakers (that is, portions of the liquid which are strongly involuted by the turbulent liquid-oxygen interaction). Oxygen absorption at the liquid surface does not limit the process of enrichment because it is particularly fast due to a very strong affinity of oxygen to the liquid iron. The thickness of the oxygen-rich region is $\sim v / v_{*}$, which is the size of the smallest eddies in the liquid, typically $\lesssim 1 \mu \mathrm{m}$ (the corresponding eddies in the gas flow are of much larger size $v_{0} / v_{*}$ ). It is only at this scale that the ordinary oxygen diffusion acts by assisting the saturation of the smallest eddies with oxygen. In fact,

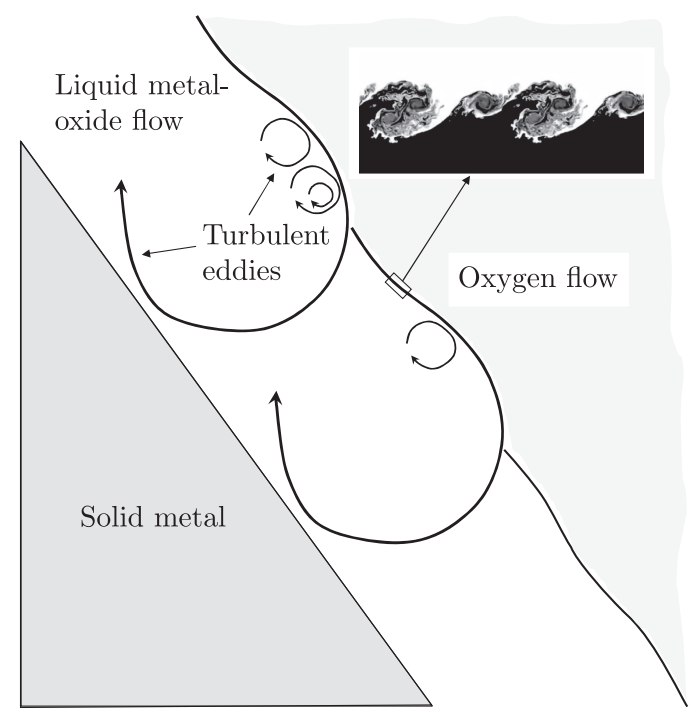

FIG. 14. Schematics of the turbulent oxygen diffusion in the liquid metal in the regime of dynamic combustion. 
since $v$ and $D$ are of the same order, the characteristic diffusion length (the penetration depth) during the smallest eddy turnover time, $v / v_{*}^{2}$, is $l_{D}=\sqrt{D v / v_{*}^{2}} \approx v / v_{*}$, which is just the smallest eddy size. This oxygen is continuously transported from the surface layer into the bulk by the larger eddies, most efficiently by the ones of the largest size $h$.

Thus, the metal oxidation rate during dynamic combustion is controlled by the rate of the turbulent mixing of oxygen with the liquid at the liquid-oxygen interface, which in turn is determined by the oxygen concentration and its speed.

Taking into account the thinness of the liquid layer, consideration can be simplified by replacing the mean oxygen concentration, $c$, by its average value $\bar{c}$ across the layer. Also, the rod can be replaced by a bar (with a square cross section) of the same dimension. This is possible by virtue of the turbulent nature of the liquid flow and the fact that the relation between the layer thickness and geometry of the solid-liquid interface is ultimately fixed by the overall mass conservation (this is in contrast to the laminar burning of vertical rods in the absence of oxygen flow, where the liquid tends to "climb" along the sample edges ${ }^{34,35}$ ). We thus have the following equation for the temperature distribution in the liquid in the regime of quasi-steady dynamic combustion

$$
\chi \frac{\partial^{2} T}{\partial \xi^{2}}+\frac{Q w \bar{c}}{c_{p} n}=0
$$

where the $\xi$ co-ordinate is along the normal to the solid-liquid interface, $Q$ is the heat of iron combustion, $n$ is the number density of iron atoms, and $w$ is the reaction rate. The latter can also be taken constant throughout the liquid, for the following reason. In the regime under consideration, the metal oxide fraction in the liquid mixture is relatively low-it does not exceed $20 \%$ [cf. Eq. (18) below]. The apparent activation energy of iron oxidation, $\mathcal{E}$, in this case is also comparatively small, about $30 \mathrm{~kJ}$ $\mathrm{mol}^{-1} .36$ Therefore, the characteristic temperature interval at the iron melting point $\left(T_{m}=1811 \mathrm{~K}\right)$

$$
\Delta T=\frac{R T_{m}^{2}}{\mathcal{E}} \approx 900 \mathrm{~K}
$$

is large compared to the temperature difference across the liquid layer, which is normally 100 to $500 \mathrm{~K}$, so that the corresponding variation in $w$ is negligible indeed.

The boundary conditions for $T(\xi)$ are

$$
\begin{gathered}
\left.T\right|_{\xi=0}=T_{m}, \\
\left.\frac{\partial T}{\partial \xi}\right|_{\xi=0}=\frac{u \cos \varphi}{\chi}\left(T_{m}-T_{0}+\frac{Q_{m}}{c_{p}}\right), \\
\left.\frac{\partial T}{\partial \xi}\right|_{\xi=h}=0 .
\end{gathered}
$$

Equation (13) is the expression of zero heat loss from the liquid to the atmosphere, whereas Eq. (12) is obtained by integrating the steady equation

$$
-u \frac{\partial T}{\partial x}=\chi \Delta T
$$

over the whole solid domain, and taking into account that $\left.T\right|_{x=+\infty}=T_{0}$; the temperature and its normal derivative are constant along the solid-liquid interface; and finally, the heat flux, $-\chi \rho c_{p} \partial T / \partial \xi$, has a jump at this surface equal to the mass flux of metal, $\rho u \cos \varphi$, times the heat of metal fusion, $Q_{m}$.

Solution of Eq. (10) satisfying condition (13) is

$$
T(\xi)=T_{a}-\frac{Q w \bar{c}}{2 \chi c_{p} n}(\xi-h)^{2}, \quad T_{a}=\text { const. }
$$

Condition (12) then yields

$$
\frac{u \cos \varphi}{h \chi}\left(T_{m}-T_{0}+\frac{Q_{m}}{c_{p}}\right)=\frac{Q w \bar{c}}{\chi c_{p} n},
$$

while condition (11) gives the temperature at the liquid surface, $T_{a}$,- - the apparent temperature of the upper rod end

$$
T_{a}=T_{m}+\frac{h u \cos \varphi}{2 \chi}\left(T_{m}-T_{0}+\frac{Q_{m}}{c_{p}}\right) .
$$

An important consequence of relation (16) is the expression for the metal oxide fraction in the liquid, $f$, which can be found as the ratio of the iron oxidation rate, $\bar{c} w h d^{2} / \cos \varphi$, to the total iron consumption rate, $u d^{2} n$. It follows from Eq. (16) that

$$
f=\frac{Q_{m}+c_{p}\left(T_{m}-T_{0}\right)}{Q} .
$$

For iron, $Q_{m}=250 \mathrm{~J} \mathrm{~g}^{-1}, Q=6000 \mathrm{~J} \mathrm{~g}^{-1}$, and $c_{p}=0.45 \mathrm{~J}$ $(\mathrm{g} \mathrm{K})^{-1}$ so that $f=15.5 \%$ for $T_{0}=300 \mathrm{~K}$.

It is to be noted that the meaning of Eq. (18) is essentially integral, in that the value of $f$ is independent of the actual oxygen distribution across the liquid. In fact, for an arbitrary $c(\xi)$, it is readily found from Eqs. (10) and (13) that

$$
\frac{\partial T}{\partial \xi}=\frac{Q w}{\chi c_{p} n} \int_{\xi}^{h} d \xi^{\prime} c\left(\xi^{\prime}\right)
$$

Equation (12) then again yields Eq. (16) in which now

$$
\bar{c}=\frac{1}{h} \int_{0}^{h} d \xi c(\xi)
$$

and relation (18) follows as before.

At this point, it is worthwhile to further discuss the possible influence of the carbon oxidation on the combustion process. Reaction

$$
\mathrm{FeO}+\mathrm{C} \rightarrow \mathrm{CO}+\mathrm{Fe}
$$

taking place in the liquid along with the iron oxidation is well known because of its importance in the oxygen steel making. It results in a reduction of $\mathrm{FeO}$ according to the fraction of carbon in the liquid. Now, the mild steel used in our experiments has less than $1 \%$ of carbon, whereas the fraction of iron oxidized during dynamic combustion is $f=15.5 \%$, as we just saw. Thus, although the fraction of iron that is actually burned is comparatively small, it is still much larger than that subjected to the process (19). Because of this smallness, the resulting changes in the fraction of iron oxide and in the heat release can be neglected. Furthermore, the $\mathrm{CO}$ gas released in the process is constantly removed by the oxygen flow, and therefore it does not affect the oxidation process. This situation can be contrasted to that in the laser oxygen cutting where even a small fraction of impurities in the oxygen flow can affect the process by accumulating from the atmosphere on the metal 
surface. To conclude, $\mathrm{CO}$ production affects neither static nor dynamic combustion of mild steel.

\section{Structure of the liquid flow near the solid-liquid interface}

As was discussed above, the liquid metal/oxide flow is essentially turbulent. Yet, it must be laminar in an immediate proximity of the solid-liquid interface because of the conditions existing thereat. This fact is important as it allows one to relate the interface slope to its propagation speed. The solid metal moves uniformly upward (with the speed $u$ in the rest frame of reference of the interface), while the liquid velocity at the interface is uniquely determined by the conservation of mass and momentum. Namely, the velocity component normal to the interface increases by the factor of $\theta=\rho / \rho_{l}$, whereas the tangential component is continuous. In the system of Cartesian co-ordinates $(\eta, \xi)$ [see Fig. 13], this gives for the liquid velocity $\boldsymbol{v}=\left(v_{\eta}, v_{\xi}\right)$

$$
v_{\eta}=-u \sin \varphi, \quad v_{\xi}=\theta u \cos \varphi \quad \text { at } \quad \xi=0 .
$$

These relations represent boundary conditions for the turbulent flow in the bulk of the liquid. This flow is governed by the continuity and Navier-Stokes equations

$$
\begin{gathered}
\operatorname{div} \boldsymbol{v}=0, \\
\frac{\partial \boldsymbol{v}}{\partial t}+(\boldsymbol{v} \boldsymbol{\nabla}) \boldsymbol{v}=-\frac{1}{\rho_{l}} \boldsymbol{\nabla} p+\boldsymbol{g}+v \Delta \boldsymbol{v} .
\end{gathered}
$$

Let $\omega$ denote the mean vorticity established, through the viscous drag, in the liquid by the external oxygen flow, $\omega$ $=\langle\operatorname{rot} \boldsymbol{v}\rangle$. In the steady-on-average flow, this quantity is constant throughout the liquid layer. This follows from the conservation of velocity circulation and the fact that any nonuniformity in $\omega$ decays due to internal friction. In particular, it has the same value near the solid-liquid interface where the flow is truly two-dimensional. If, in addition to that, it is truly steady there, it obeys

$$
\begin{gathered}
\frac{\partial v_{\eta}}{\partial \eta}+\frac{\partial v_{\xi}}{\partial \xi}=0, \quad \frac{\partial v_{\xi}}{\partial \eta}-\frac{\partial v_{\eta}}{\partial \xi}=\omega, \\
v_{\eta} \frac{\partial v_{\eta}}{\partial \eta}+v_{\xi} \frac{\partial v_{\eta}}{\partial \xi}=-\frac{1}{\rho_{l}} \frac{\partial p}{\partial \eta}+g \sin \varphi+v \Delta v_{\eta}, \\
v_{\eta} \frac{\partial v_{\xi}}{\partial \eta}+v_{\xi} \frac{\partial v_{\xi}}{\partial \xi}=-\frac{1}{\rho_{l}} \frac{\partial p}{\partial \xi}-g \cos \varphi+v \Delta v_{\xi},
\end{gathered}
$$

where $\omega$ is the given constant value of $\omega=(0,0, \omega)$, and $p$ is the liquid pressure. An obvious solution of this system reads

$$
v_{\eta}=-\omega \xi+\text { const }, \quad v_{\xi}=-\frac{g \sin \varphi}{\omega}, \quad p=-\xi \rho_{l} g \cos \varphi .
$$

It can be shown that this solution is unique under the condition of vanishing pressure gradient along the solid-liquid interface (unrestricted liquid flow). As was mentioned, it is valid only in a vicinity of this interface where the liquid flow is laminar. Now, the first of the boundary conditions (20) gives const $=-u \sin \varphi$, while the second leads to the following relation between the inclination angle, induced vorticity, and the speed of rod burning

$$
\tan \varphi=-\frac{\omega \theta u}{g}
$$

It is to be noted that the solid density change due to the rod preheat is not to be taken into account in this formula: the product $\rho u$ is constant by virtue of the same mass-flux conservation that led to the appearance of the factor $\theta$ in Eq. (27); on the other hand, as defined, $u$ is also the speed of the upper rod end with respect to its lower end, that is, the cold metal. Therefore, $\theta=\rho / \rho_{l}$ is to be calculated with $\rho$ at $T=T_{0}$. At the same time, $\rho_{l}$ is to be taken at $T=T_{m}$, as Eq. (27) is a consequence of the flow equations near the solid-liquid interface.

Taking the run shown in Fig. 10 as an example, substitution of $d=0.3 \mathrm{~cm}, v_{0}=0.15 \mathrm{~cm}^{2} \mathrm{~s}^{-1}$, and $v_{0}=10 \mathrm{~m} \mathrm{~s}^{-1}$ into Eq. (6) gives $v_{*}=120 \mathrm{~cm} \mathrm{~s}^{-1}$. With $\eta=3.5 \cdot 10^{-2} \mathrm{~g} \mathrm{~cm}^{-1} \mathrm{~s}^{-1}$, $\rho_{0}=1.2 \cdot 10^{-3} \mathrm{~g} \mathrm{~cm}^{-3}$, we then find $\omega=-\rho_{0} v_{*}^{2} / \eta \approx-500 \mathrm{~s}^{-1}$. Inserting this together with $\theta=1.12$ and the value $u=1 \mathrm{~cm}$ $\mathrm{s}^{-1}$ measured on the interval $500 \mathrm{~ms}$ to $700 \mathrm{~ms}$ into formula (27), one finds $\tan \varphi=0.57$ or $\varphi=30^{\circ}$.

It should be stressed that in addition to the conditions identified in Sec. IV C 1, derivation of Eq. (27) assumes that the flow near the solid-liquid interface is steady. While the steadiness-on-average of the bulk liquid flow is a natural consequence of its comparatively fast (turbulent) dynamics, so that it is readily established at any given $\varphi \neq 0$, the flow near the interface may or may not be truly steady. Our observations show that the inclination angle monotonically increases in time, with a more or less extended plateau which presumably corresponds to the steadiness condition at the interface. To clarify this point would apparently require a joint stability analysis of the heat and mass transfer near the interface.

\section{Oxygen concentration in the liquid layer}

Oxygen transport in the liquid layer is described by the following equation for the concentration of oxygen atoms, $C$ :

$$
\frac{\partial C}{\partial t}+(\boldsymbol{v} \nabla) C=D \Delta C-w C .
$$

As was already noted, the ordinary diffusion term $D \Delta C$ can be omitted as it is negligible compared to the turbulent contribution. Decomposing the fluid velocity and concentration into the mean and fluctuating parts

$$
\boldsymbol{v}=\langle\boldsymbol{v}\rangle+\tilde{\boldsymbol{v}}, \quad C=c+\tilde{c}, \quad c \equiv\langle C\rangle,
$$

and recalling that $c=\bar{c}$ (see Sec. IV C 1), one finds

$$
\frac{\partial \tilde{c}}{\partial t}+(\langle\boldsymbol{v}\rangle \boldsymbol{\nabla}) \tilde{c}+(\tilde{\boldsymbol{v}} \boldsymbol{\nabla}) \tilde{c}=-w(\bar{c}+\tilde{c}) .
$$

Statistical averaging of this equation gives

$$
\langle(\tilde{\boldsymbol{v}} \boldsymbol{\nabla}) \tilde{c}\rangle=-w \bar{c} .
$$

According to the definition of $\bar{c}$, the left hand side is to be further averaged over the volume $V$ of the liquid layer. Rewriting the volume integral as a surface integral with the help of the flow continuity ( $\operatorname{div} \tilde{\boldsymbol{v}}=0$ ), we obtain

$$
\langle(\tilde{\boldsymbol{v}} \boldsymbol{\nabla}) \tilde{c}\rangle=\frac{1}{V} \int_{V} d V(\tilde{\boldsymbol{v}} \boldsymbol{\nabla}) \tilde{c}=\frac{1}{V} \int_{S}(d s \tilde{\boldsymbol{v}}) \tilde{c},
$$

where $d s$ is the element of the layer surface $S$, directed outwards of the liquid. Whenever a turbulent fluctuation shifts the local surface position towards the liquid (as in Fig. 15), that is, $(d s \tilde{\boldsymbol{v}})<0$, the fluctuation in the oxygen concentration brought in thereby equals to $\left(2 c_{0}-\bar{c}\right)$, while for $(d s \tilde{\boldsymbol{v}})>0$, 


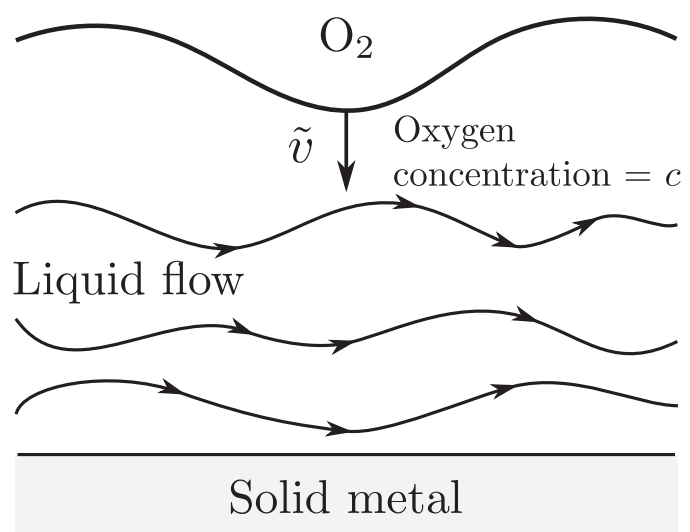

FIG. 15. Schematics of the enrichment of the liquid metal with oxygen used in evaluating the integral on the right of Eq. (29). Shown is a fluctuation of the liquid-gas interface produced by an eddy of smallest size $v / v_{*}$, where $\tilde{\boldsymbol{v}}$ denotes a velocity fluctuation of the order $v_{*}$.

the fluctuation $\tilde{c}=\left(\bar{c}-2 c_{0}\right)$. Here $c_{0}$ is the concentration of oxygen molecules in the ambient gas flow, and the factor of two accounts for the chemical adsorption of oxygen atoms. Neglecting $\bar{c}$ in comparison with $c_{0}$, we thus have

$$
\int_{S}(d s \tilde{\boldsymbol{v}}) \tilde{c}=-2 c_{0} \int_{S} d s\left|\tilde{v}_{n}\right|,
$$

where $\tilde{v}_{n}$ is the normal to the surface component of fluctuating liquid velocity. By virtue of the boundary conditions at the liquid-gas interface, this is also the normal velocity component of the gas flow. Substituting this in Eq. (29), writing $V$ $=h S$, and putting the result in Eq. (28) yield the mean oxygen concentration in the liquid

$$
\bar{c}=\frac{2 c_{0} v_{*}}{w h}, \quad v_{*}=\frac{1}{S} \int_{S} d s\left|\tilde{v}_{n}\right| .
$$

The quantity $\frac{1}{S} \int_{S} d s\left|\tilde{v}_{n}\right|$ appearing in this formula is denoted $v_{*}$ because it is the velocity fluctuation averaged over the liquid-gas interface, which is nothing but an estimate of the friction velocity [introduction of an order-of-unity proportionality coefficient in this relation would amount merely to a redefinition of that in the law (6), which itself is well-known to be nonunique; the value 2.5 (corresponding to the "canonical" value 0.4 of the Kármán constant) is used in this law because it yields satisfactory fit to the experimental data, see Sec. V below].

Although the above integral calculation is a direct expression of the idea of turbulent transport, it is perhaps worth to relate it to the more conventional differential formulation. In the latter, the average $\langle\tilde{\boldsymbol{v}} \tilde{c}\rangle$ is postulated to be proportional to the mean concentration gradient,

$$
\langle\tilde{\boldsymbol{v}} \tilde{c}\rangle=-D_{t} \boldsymbol{\nabla} c,
$$

where $D_{t}$ is the coefficient of turbulent diffusion. The right hand side of Eq. (29) then becomes

$$
-\frac{D_{t}}{V} \int_{S}(d \boldsymbol{s} \nabla c)=-\left.\frac{D_{t}}{h} \frac{\partial c}{\partial \xi}\right|_{\xi=h} .
$$

The mean concentration increases from zero to $2 c_{0}$ between the solid-liquid and liquid-gas interfaces. Therefore, estimating the concentration gradient by $2 c_{0} / h$, Eq. (28) gives

$$
\bar{c}=\frac{2 c_{0} D_{t}}{w h^{2}},
$$

and comparison with Eq. (30) yields an expression for the turbulent diffusion coefficient

$$
D_{t}=v_{*} h .
$$

This expression is obviously in accordance with the view on the oxygen diffusion as a large-eddy transport of local fluctuations in the oxygen concentration which are caused by the turbulent velocity pulsations of the order $v_{*}$ at the liquid-gas interface.

a. Normal speed of metal burning. The obtained results allow us to express the normal speed of the solid-liquid interface as a function of the imposed flow conditions. By combining Eq. (30) with Eq. (16), we arrive at the following formula for $u_{n}=u \cos \varphi$ :

$$
u_{n}=v_{*} \frac{2 c_{0}}{f n},
$$

where $f$ is given by Eq. (18). In analogy with premixed combustion of gases, this quantity can be called the local rate of metal burning, though the actual volumetric rate of metal oxidation is $f u_{n}$, while the number of iron atoms oxidized per unit time per unit area of the solid-liquid interface is

$$
f n u_{n}=2 v_{*} c_{0} .
$$

As an example, under normal pressure and temperature, $c_{0}=2.4 \times 10^{19} \mathrm{~cm}^{-3}$, whereas the density of atoms in the liquid iron $n=7.4 \times 10^{22} \mathrm{~cm}^{-3}$ so that $c_{0} / n=0.32 \times 10^{-3}$; in the case of Fig. $10\left(v_{0}=10 \mathrm{~m} \mathrm{~s}^{-1}, v_{*}=120 \mathrm{~cm} \mathrm{~s}^{-1}\right)$, Eq. (33) yields $u_{n}=0.5 \mathrm{~cm} \mathrm{~s}^{-1}$.

$b$. Normal speed in the absence of oxygen flow. Since the oxygen gas density is proportional to its pressure, $c_{0} \sim p_{0}$, a self-sustained propagation of the reaction is possible at elevated oxygen pressures, $p_{0}$, even in the absence of an oxygen flow. The propagation speed in this case is lower, as the liquid velocity fluctuations at the liquid-gas interface are now only due to the turbulent motion caused by the decaying baroclinic vorticity, $\left|\tilde{v}_{n}\right| \approx \omega_{g} h$. Substituting this for $v_{*}$ in Eq. (33) and using Eq. (5) give an estimate for the normal speed

$$
u_{n} \approx \frac{\theta-1}{\theta} \frac{g \sin \varphi}{u_{n}} \frac{2 c_{0} h}{f n}
$$

or

$$
u_{n} \approx \sqrt{\frac{\theta-1}{\theta} \frac{2 c_{0} h g \sin \varphi}{f n}} .
$$

Thus, the normal speed of self-sustained propagation $\sim p_{0}^{1 / 2}$. It is worth recalling in this connection that the same dependence on the oxygen pressure is found when the chemical adsorption $\left(\mathrm{O}_{2} \rightarrow \mathrm{O}+\mathrm{O}\right)$ of oxygen atoms at the metal surface is the rate-determining step of the reaction. ${ }^{19}$ This circumstance might lead to confusion in experimental determination of the combustion mechanism. In our experiments, however, the normal burning speed was never less than a few millimeters per second, just as predicted by the estimate (35). It follows that the chemical adsorption does not slow down the burning process. 


\section{BURNING IN OXYGEN FLOWS UNDER ELEVATED PRESSURES}

Among the factors appearing in the expression (33) for the normal burning speed, dependence on the oxygen concentration appears to be the easiest for experimental verification. However, our experimental setup does not allow appreciable pressure changes at the oxygen flow speeds higher than $10 \mathrm{~m} \mathrm{~s}^{-1}$. Also, since we are not able to control the azimuth of the liquid metal flow with respect to the rod axis, only in a few instances it was possible to obtain a profile view of the metal flow that would allow the determination of its slope and propagation speed. These are collected in Table I, and Figure 16 gives an example of these determinations. Experimental precision of each slope or speed determination is about $1 \%-2 \%$, but in the absence of statistics for a given pair $v_{0}, p_{0}$ due to the reason just mentioned, it is difficult to give an estimate of the total experimental uncertainty. As to the calculational error, it is primarily due to the error in evaluating the friction velocity. It can be roughly estimated by noting that if one admits an error as large as a factor of two in the boundary layer thickness of the oxygen flow, then the relative error in $v_{*}$ for, say, $7 \mathrm{~m} \mathrm{~s}^{-1}$ flow speed is $\ln 2 / \ln \left(v_{*}^{2} d / v_{0} v_{0}\right) \approx 20 \%$. As is seen from Table I, the calculated and measured values for the normal burning speed agree within this accuracy.

Significantly larger pressures have been achieved for vanishing oxygen flow speed. In this case, however, the process of

TABLE I. Comparison of the calculated $\left(u_{n}\right)$ and measured $\left(u_{n}^{\exp }\right)$ normal propagation speeds of metal burning at various oxygen flow speeds and pressures.

\begin{tabular}{lcccccc}
\hline \hline $\begin{array}{l}v_{0} \\
\left(\mathrm{~m} \mathrm{~s}^{-1}\right)\end{array}$ & $p_{0}(\mathrm{~atm})$ & $\begin{array}{c}v_{*} \\
\left(\mathrm{~cm} \mathrm{~s}^{-1}\right)\end{array}$ & $\begin{array}{c}u^{\exp } \\
\left(\mathrm{cm} \mathrm{s}^{-1}\right)\end{array}$ & $\begin{array}{c}\varphi^{\exp } \\
(\mathrm{deg})\end{array}$ & $\begin{array}{c}u_{n}^{\exp } \\
\left(\mathrm{cm} \mathrm{s}^{-1}\right)\end{array}$ & $\begin{array}{c}u_{n} \\
\left(\mathrm{~cm} \mathrm{~s}^{-1}\right)\end{array}$ \\
\hline 3 & 8.5 & 45.6 & 8.5 & 78 & 1.8 & 1.7 \\
3 & 11.0 & 45.6 & 12.7 & 79 & 2.4 & 2.1 \\
7 & 6.0 & 89.5 & 14.6 & 80 & 2.4 & 2.3 \\
7 & 8.5 & 89.5 & 21.3 & 81 & 3.4 & 3.2 \\
7 & 11.0 & 89.5 & 19.0 & 77 & 4.3 & 4.2 \\
\hline \hline
\end{tabular}

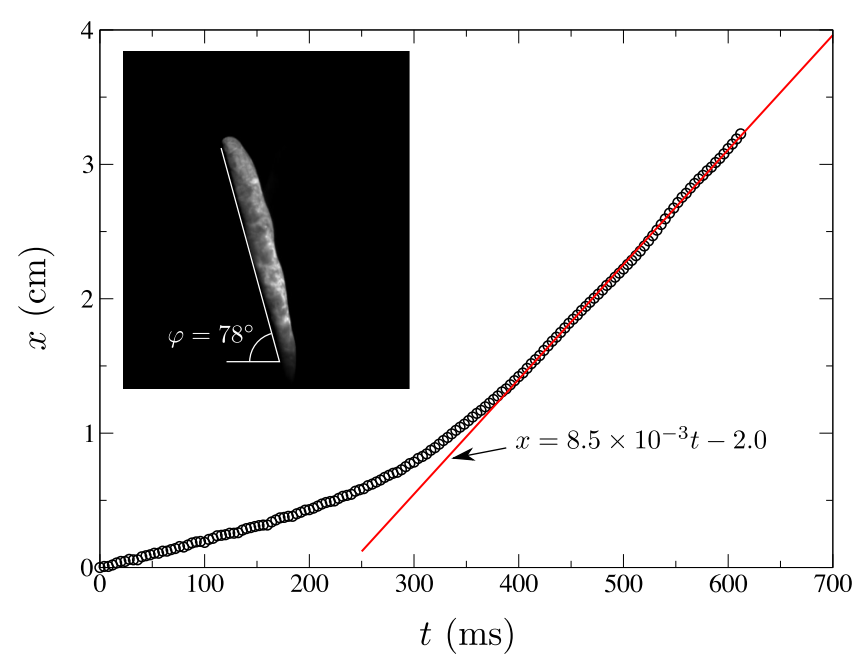

FIG. 16. Measured position of the upper rod end (marks) and its linear approximation (solid line) for oxygen speed and pressure of $3 \mathrm{~m} \mathrm{~s}^{-1}$ and $8.5 \mathrm{~atm}$, respectively. Inset: determination of the solid-liquid interface slope.

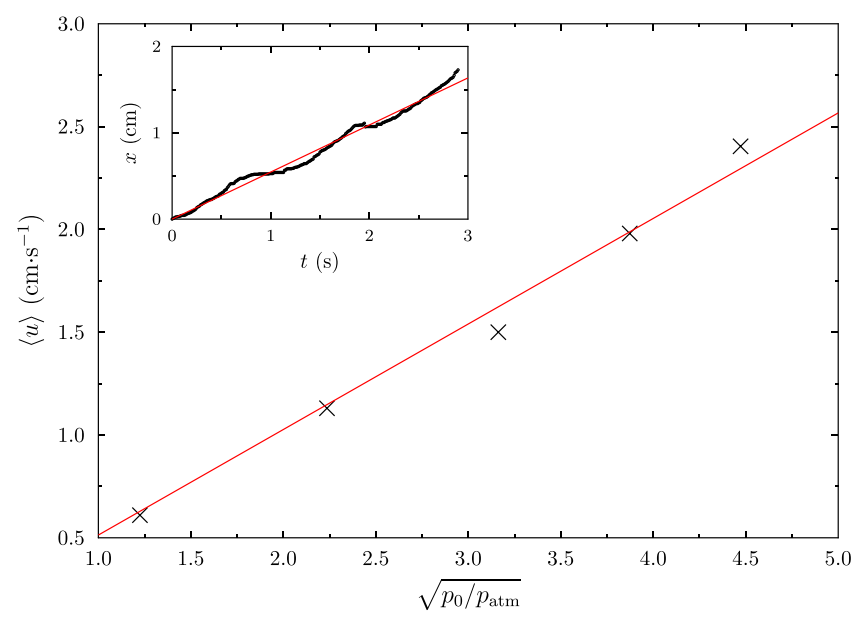

FIG. 17. The time-averaged vertical speed of the upper rod end against oxygen pressure. Marks represent measured values, line-best fit $\langle u\rangle$ $=0.49\left(p_{0} / p_{\text {atm }}\right)^{0.52} \cdot p_{\text {atm }}$ denotes normal pressure. Inset: the rod end trajectory in the case $p_{0}=1.5 \mathrm{~atm}$.

rod regression is more complicated-it is a periodic sequence of the liquid cap swells and falls along the solid-liquid interface whose inclination is likewise periodic. This periodicity is illustrated in the inset of Fig. 17 that shows the rod end trajectory. In addition to that, the liquid cap azimuthal motions are even less regular than in the presence of an oxygen flow. Yet, this behavior does not preclude completely an experimental verification of Eq. (35). To test the predicted $p_{0}^{1 / 2}$-dependence of the propagation speed, which is of major interest, we average $u=u_{n} / \cos \varphi$ over time (denoting this operation by angular brackets)

$$
\left.\langle u\rangle \approx \sqrt{\frac{2(\theta-1) c_{0} g}{\theta f n}} \cdot \mid \sqrt{\frac{h \sin \varphi}{\cos ^{2} \varphi}}\right) .
$$

In the absence of oxygen flow, the liquid cap geometry is controlled by the gravity and surface tension. Therefore, the last factor in this expression can be taken as a constant independent of the oxygen pressure (it can be noted also that the periodic variations in this term are smaller than the individual variations in $h$ and $\varphi$, because the cap thickness grows whenever the inclination angle decreases so that the two variations partially cancel each other). The measured dependence $\langle u\rangle\left(\sqrt{p_{0}}\right)$ is plotted in Fig. 17. It is also of interest to make an order-ofmagnitude comparison of the measured and calculated proportionality coefficients in the relation $\langle u\rangle \sim \sqrt{p_{0} / p_{\text {atm }}}$ [derivation of Eq. (35) does not permit any more accurate evaluation]. According to the discussion in Sec. IV B, one can expect that $\langle\sqrt{h \sin \varphi} / \cos \varphi\rangle \sim \sqrt{d}$, and the video images of the process show that the proportionality symbol $\sim$ can be replaced (within the order-of-magnitude accuracy) with an approximate equality. For $d=3 \mathrm{~mm}$, Eq. (36) thus gives $\langle u\rangle \approx 0.4 \mathrm{~cm} \mathrm{~s}^{-1}$ at $p_{0}$ $=p_{\text {atm }}$, whereas the experimental value is $\langle u\rangle \approx 0.5 \mathrm{~cm} \mathrm{~s}^{-1}$.

\section{DISCUSSION AND CONCLUSIONS}

The results of our study show that the process of iron and mild steel combustion is strongly affected by the motions of ambient oxygen atmosphere: it turns out to be driven by an axial oxygen flow already at the flow speeds of a few meters 
per second. The main reason for this dramatic change in the combustion regime as compared to the burning in a still atmosphere is the changeover of the oxygen transport through the liquid metal from ordinary to turbulent diffusion. The smallscale turbulent motions at the liquid-gas interface enrich the melt with oxygen which is then rapidly redistributed over the whole liquid by larger eddies. The rate of metal consumption is thus controlled by the rate of this enrichment, which in turn is determined by the oxygen concentration and its speed. This is quantified by Eq. (33) for the normal speed of the solidliquid interface: it is proportional to the oxygen concentration, and up to a logarithmic factor, to its speed. It is also naturally in inverse proportionality to the fraction of the metal oxide: the larger this fraction, the lesser the rate of metal melting is required to sustain a steady regime of metal combustion. It is worth to stress in this connection that the process of oxygen enrichment by the turbulent motions at the liquid-gas interface is autonomous in that it is independent of the metal consumption rate. It is by this reason that it turned out to be possible to express the normal speed as a function of the oxygen concentration and its flow speed. This situation resembles that found in premixed flames with high activation energy of the reaction, in which case the reaction zone within the flame front is well-known to acquire similar independence of the flame propagation speed.

The quantitative agreement of the calculated and measured normal speeds of the solid-liquid interface allows us to conclude that the theoretical approach developed in Sec. IV C adequately describes the physics of iron combustion in oxygen flows.

Formula (18) for the oxide fraction itself deserves further discussion. The point is that this formula does not involve the vertical speed $u$ of the interface or any other dynamical characteristic of the sample burning. This suggests that its validity is not limited by the quasi-steady condition. Indeed, it is merely an expression of the fact that the front of combustion - the solid-liquid interface-propagates at a constant temperature (the metal melting point), and Eq. (18) just states that $(f Q)$ is the heat needed to preheat and melt a unit mass of metal. If, for some reason, the actual fraction would exceed $f$, an increased heat flux to the solid region would increase the speed of the interface advancement, bringing more metal into the liquid flow and reducing thereby the oxide fraction.

The fact that under the given oxygen-flow conditions, the normal speed of the solid-liquid interface is a constant independent of the interface inclination implies that the vertical speed of the interface is proportional to the interface area, $S$. Indeed, $u=u_{n} / \cos \varphi=u_{n} 4 S /\left(\pi d^{2}\right)$. This is exactly the conclusion of Refs. 37 and 38 that studied the effect of gravity on iron burning (without oxygen flow). Although the normal speeds in the two cases are quite different, this suggests that the existence of a well-defined normal speed (specific to the given conditions) is a general property of iron combustion. Once inferred, it can be used to model iron burning with more complex interface geometry. Typical applied problems would be the evolution of a hole in an oxygen pipeline or of a small hole in a high-pressure oxygen container. In the latter case, the oxygen flow itself is caused by the growing hole so that it will have to be consistently determined together with the hole size and its shape.

\section{ACKNOWLEDGMENTS}

K. Kazakov is grateful to the French Government for supporting his stay at Poitiers University during the fall of 2012 under the program "Investissements d'Avenir" (LABEX INTERACTIFS, Reference No. ANR-11-LABX-0017-01). The authors also thank Professor R. Fabbro for discussions.

${ }^{1}$ F. Maggi, G. Gariani, L. Galfetti, and L. T. DeLuca, "Theoretical analysis of hydrides in solid and hybrid rocket propulsion," Int. J. Hydrogen Energy 37(2), 1760 (2012).

${ }^{2}$ D. R. Greatrix, "Multisized inert particle loading for solid rocket axial combustion instability suppression," Int. J. Aerosp. Eng. 2012, 173129 (2012).

${ }^{3}$ A. G. Merzhanov, "History and recent developments in SHS," Ceram. Int. 21(5), 371 (1995).

${ }^{4}$ Self-propagating High-temperature Synthesis of Materials, edited by A. A. Borisov, L. De Luca, and A. Merzhanov (Taylor \& Francis, New York, USA, 2002).

${ }^{5}$ R. A. Yetter, G. A. Risha, and S. F. Son, "Metal particle combustion and nanotechnology," Proc. Combust. Inst. 32(2), 1819 (2009).

${ }^{6}$ A. E. Sytschev, S. G. Vadchenko, O. D. Boyarchenko, D. Vrel, and N. V. Sachkova, "SHS joining of intermetallics with metallic substrates," Int. J. Self-Propag. High-Temp. Synth. 20(3), 185 (2011).

${ }^{7}$ V. N. Sanin, D. M. Ikornikov, N. V. Sachkova, and V. I. Yukhvid, "Complex boride metal-matrix composites by SHS under high gravity,” Int. J. SelfPropag. High-Temp. Synth. 23(3), 151 (2014).

${ }^{8}$ C. K. Law, "A simplified theoretical model for the vapor-phase combustion of metal particles," Combust. Sci. Technol. 7(5), 197 (1973).

${ }^{9}$ S. B. Margolis, "An asymptotic theory of heterogeneous condensed combustion,” Combust. Sci. Technol. 43(3-4), 197 (1985).

${ }^{10}$ A. Makino, "Fundamental aspects of the heterogeneous flame in the self-propagating high-temperature synthesis (SHS) process," Prog. Energy Combust. Sci. 27(1), 1 (2001).

${ }^{11}$ D. Meinköhn, "Liquid oxide surface layers in metal combustion," Combust. Theory Modell. 8(2), 315 (2004).

${ }^{12}$ D. Meinköhn, "Metal-particle ignition and oxide-layer instability," Combust., Explos. Shock Waves 42(2), 158 (2006).

${ }^{13}$ D. Meinköhn, "The effect of particle size and ambient oxidizer concentration on metal particle ignition," Combust. Sci. Technol. 181(8), 1007 (2009).

${ }^{14}$ B. B. Khina, Combustion Synthesis of Advanced Materials (Nova Science Publishers, New York, USA, 2010).

${ }^{15}$ K. K. Kuo and R. Acharya, Applications of Turbulent and Multi-phase Combustion (Wiley, New Jersey, USA, 2012).

${ }^{16}$ R. A. Rhein and C. M. Carlton, "Extinction of lithium fires: Thermodynamic computations and experimental data from literature," Fire Technol. 29(2), 100 (1993).

${ }^{17}$ A. F. Clark and J. G. Hust, "A review of the compatibility of structural materials with oxygen," AIAA J. 12(4), 441 (1974).

${ }^{18}$ T. A. Steinberg, M. A. Rucker, and H. D. Beeson, in Flammability and Sensitivity of Materials in Oxygen-enriched Atmospheres, ASTM STP 1040, edited by J. Stoltzfus, J. Stradling, and F. Benz (American Society for Testing and Materials, Philadelphia, 1989), Vol. 4, pp. 54-75.

${ }^{19}$ T. Hirano, K. Sato, Y. Sato, and J. Sato, "Prediction of metal fire spread in high pressure oxygen,” Combust. Sci. Technol. 32(1-4), 137 (1983).

${ }^{20}$ S. I. Shabunya, V. V. Martynenko, V. I. Ignatenko, and J.-Ch. Rostaing, "Simulation of the combustion of thin iron rods in oxygen in the adiabatic approximation," J. Eng. Phys. Thermophys. 87(6), 1279 (2014).

${ }^{21}$ T. A. Steinberg and F. J. Benz, Flammability and Sensitivity of Material in Oxygen-Enriched Atmospheres, ASTM STP1111, edited by J. Stoltzfus and K. McIlroy (American Society for Testing and Materials, Philadelphia, 1991), Vol. 5, pp. 298-312.

${ }^{22}$ J. Sato, H. Ohtani, and T. Hirano, "Ignition process of a heated iron block in high-pressure oxygen atmosphere," Combust. Flame 100(3), 376 (1995).

${ }^{23}$ M. Muller, R. Fabbro, H. El-Rabii, and K. Hirano, "Temperature measurement of laser heated metals in highly oxidizing environment using 2D single-band and spectral pyrometry," J. Laser Appl. 24(2), 022006 (2012). 
${ }^{24}$ M. Muller, H. El-Rabii, and R. Fabbro, "Laser ignition of bulk iron, mild steel, and stainless steel in oxygen atmospheres," Combust. Sci. Technol. 186(7), 953 (2014).

${ }^{25}$ CRC Handbook of Chemistry and Physics, edited by D. R. Lide (CRC Press, Boca Raton, FL, 2006).

${ }^{26}$ G. Shen, P. Lazor, and S. K. Saxena, "Melting of wüstite and iron up to pressures of 600 kbar,” Phys. Chem. Miner. 20(2), 91 (1993).

${ }^{27}$ M. Muller, H. El-Rabii, and R. Fabbro, "Liquid phase combustion of iron in an oxygen atmosphere," J. Mater. Sci. 50(9), 3337 (2015).

${ }^{28}$ The temperature peaks seen in the figures do represent the droplet surface temperature, not just a momentary increase in radiation due to its increased surface area, as the temperature falls down well before the droplet slides down the rod.

${ }^{29}$ W. D. Hayes, "The vorticity jump across a gasdynamic discontinuity," J. Fluid Mech. 2(6), 595 (1957).

${ }^{30}$ H. Schlichting, Boundary-layer Theory (McGraw-Hill, New York, USA, 1979).

${ }^{31}$ M. J. Assael, K. Kakosimos, R. M. Banish, J. Brillo, I. Egry, R. Brooks, P. N. Quested, K. C. Mills, A. Nagashima, Y. Sato, and W. A. Wakeham,
"Reference data for the density and viscosity of liquid aluminum and liquid iron,” J. Phys. Chem. Ref. Data 35(1), 285 (2006).

${ }^{32}$ I. Miyamoto and H. Maruo, "Mechanism of laser cutting," Weld. World 29(9), 283 (1991).

${ }^{33}$ Y. Li, R. J. Fruehan, J. A. Lucas, and G. R. Belton, "The chemical diffusivity of oxygen in liquid iron oxide and a calcium ferrite," Metall. Mater. Trans. B 31(5), 1059 (2000).

${ }^{34}$ T. Suvorovs, N. Ward, R. Wilson, and T. Steinberg, "Effect of sample geometry on regression rate of the melting interface for carbon steel burned in oxygen," J. ASTM Int. 3(4), 13565 (2006).

${ }^{35}$ T. Suvorovs, N. R. Ward, T. A. Steinberg, and R. Wilson, "Effect of geometry on the melting rates of iron rods burning in high pressure oxygen," J. ASTM Int. 4(4), 101004 (2007).

${ }^{36}$ D. Gamburg and B. Sarychev, Tr. GIAP 7, 121 (1957).

${ }^{37}$ N. R. Ward and T. A. Steinberg, "Iron burning in pressurised oxygen under microgravity conditions," Microgravity Sci. Technol. 21(1), 41 (2009).

${ }^{38}$ N. R. Ward and T. A. Steinberg, "A proposed qualitative framework for heterogeneous burning of metallic materials: The "melting rate triangle,," J. ASTM Int. 6(6), 102270 (2009). 